\section{Digital Beamforming on}

Receive: Techniques and Optimization Strategies for High-Resolution Wide-Swath SAR Imaging

\author{
NICOLAS GEBERT \\ GERHARD KRIEGER, Member, IEEE \\ ALBERTO MOREIRA, Fellow, IEEE \\ German Aerospace Center (DLR)
}

Synthetic Aperture Radar (SAR) is a well-proven imaging technique for remote sensing of the Earth. However, conventional SAR systems are not capable of fulfilling the increasing demands for improved spatial resolution and wider swath coverage. To overcome these inherent limitations, several innovative techniques have been suggested which employ multiple receive-apertures to gather additional information along the synthetic aperture. These digital beamforming (DBF) on receive techniques are reviewed with particular emphasis on the multi-aperture signal processing in azimuth and a multi-aperture reconstruction algorithm is presented that allows for the unambiguous recovery of the Doppler spectrum. The impact of Doppler aliasing is investigated and an analytic expression for the residual azimuth ambiguities is derived. Further, the influence of the processing on the signal-to-noise ratio (SNR) is analyzed, resulting in a pulse repetition frequency (PRF) dependent factor describing the SNR scaling of the multi-aperture beamforming network. The focus is then turned to a complete high-resolution wide-swath SAR system design example which demonstrates the intricate connection between multi-aperture azimuth processing and the system architecture. In this regard, alternative processing approaches are compared with the multi-aperture reconstruction algorithm. In a next step, optimization strategies are discussed as pattern tapering, prebeamshaping-on-receive, and modified processing algorithms. In this context, the analytic expressions for both the residual ambiguities and the SNR scaling factor are generalized to cascaded beamforming networks. The suggested techniques can moreover be extended in many ways. Examples discussed are a combination with ScanSAR burst mode operation and the transfer to multistatic sparse array configurations.

Manuscript received January 29, 2007; revised May 16, 2007; released for publication March 25, 2008.

IEEE Log No. T-AES/45/2/933006.

Refereeing of this contribution was handled by V. Chen.

This work was supported by EADS Defence Electronics, Ulm, Germany.

Authors' address: German Aerospace Center (DLR), Microwaves and Radar Institute (HR), Muenchener Strasse 20, Wessling, 82230, Germany, E-mail: (nico.gebert@dlr.de).

0018-9251/09/\$25.00 (c) 2009 IEEE

\section{INTRODUCTION}

Remote sensing of the Earth's surface demands sensors that are capable of continuous global coverage and, in addition, provide detailed imagery. Important applications are e.g., disaster management, land and sea traffic observation, wide area surveillance, and environmental monitoring. Conventional synthetic aperture radar (SAR) systems cannot meet these rising demands as the unambiguous swath width and the achievable azimuth resolution pose contradicting requirements on system design [1]. A good azimuth resolution $\delta_{a z}$ requires a high Doppler bandwidth $B_{D}$ that results from a long synthetic aperture which is illuminated by a short antenna of length $L_{a}$ (cf. (1)). Hence, a high pulse repetition frequency (PRF) is needed to sample the Doppler spectrum according to the Nyquist criterion. In contrast, to unambiguously image a wide swath of width $W_{g}$ on ground, a large interval between subsequent pulses is favorable what corresponds to a low PRF (cf. (2))

$$
\begin{aligned}
& \delta_{a z} \approx \frac{L_{a}}{2} \approx \frac{v_{s}}{B_{D}} \geq \frac{v_{s}}{\mathrm{PRF}} \\
& W_{g}<\frac{c}{2 \cdot \mathrm{PRF} \cdot \sin \left(\Theta_{i}\right)}
\end{aligned}
$$

where $\Theta_{i}$ represents the incident angle, $v_{s}$ the sensor velocity, and $c$ the speed of light. To obtain a figure of merit that combines swath width and resolution, the reciprocal of (1) is multiplied with (2). This yields expression (3) that does not depend any more on adjustable system parameters, which means that the SAR parameters underlie a trade-off, as the resolution can only be enhanced at the cost of a decreased swath width and vice versa

$$
\frac{W_{g}}{\delta_{a z}}<\frac{c}{2 \cdot v_{s} \cdot \sin \left(\Theta_{i}\right)} .
$$

Alternative SAR imaging modes push this trade-off only further into one direction or another without resolving the underlying system-inherent constraint: the spotlight mode yields a high resolution, but no sufficient coverage [2] while burst modes as ScanSAR $[3,4]$ and TOPS-SAR [5] map a wide swath but provide only a coarse resolution.

Consequently, new system concepts are needed to fulfill the increasing demands of future SAR missions. The most promising concepts employ multi-channel SAR systems where the receiving antenna is either split into multiple subapertures with independent receiver channels or the receiver apertures are distributed on multiple platforms leading to a multistatic SAR. References [6]-[37] list the various approaches in chronological order. All methods are based on the simultaneous reception of the backscattered signal with mutually displaced receiving apertures. The additional information permits to overcome the aforementioned inherent 


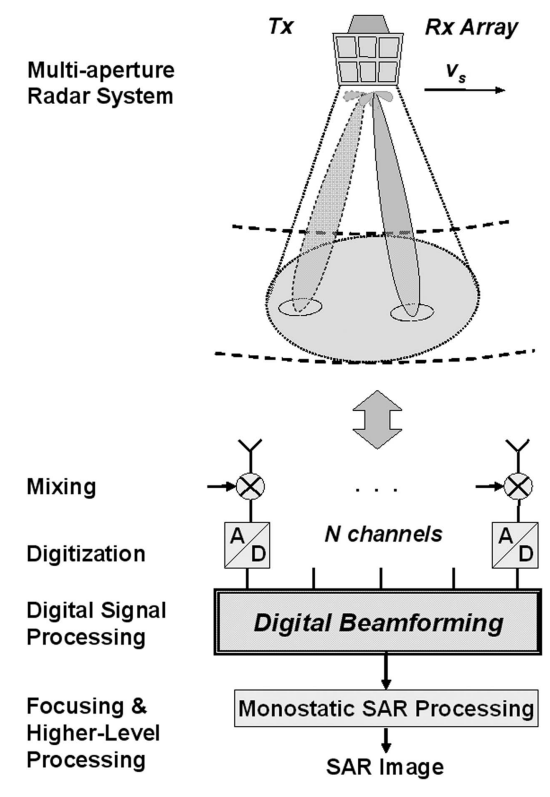

Fig. 1. Multi-aperture system with formation of different beams (top) and corresponding block diagram with DBF on receive principle (bottom). Each aperture is interpreted as individual channel whose signals are digitized and stored before combined coherently a posteriori.

limitations of conventional SAR systems. The basic concept of a multi-aperture SAR system is illustrated in Fig. 1. Each of the $N$ receiver channel's signals is mixed, digitized, and stored. Then, a posteriori, digital beamforming (DBF) on receive is carried out by a joint spatiotemporal processing of the recorded subaperture signals. In azimuth dimension this enables a coherent combination of the $N$ subsampled and hence aliased signals to a single output signal that is sampled with $N \cdot$ PRF and free of aliasing. Compared with a mono-aperture system operated with PRF, the additional samples increase the effective sampling by a factor of $N$ and allow either for an improved resolution or a reduction of the PRF without an increase of azimuth ambiguities, thereby enabling the mapping of a wide swath.

In the following, a brief overview over different multi-aperture systems and processing strategies is given. More details can be found in the corresponding references. Concerning classical side-looking multi-aperture SAR systems, the development started with proposing an array antenna in elevation to suppress range ambiguous returns [9] and the idea of splitting the antenna in azimuth direction to reduce azimuth ambiguities [10] followed by the combination of both [11]. Then, the complexity of the systems increased and interest turned to more sophisticated processing strategies enabling so-called "software-defined radars," where the multi-aperture SAR signal is processed in azimuth, elevation, or both dimensions [13-17, 20-23].

Regarding the azimuth dimension, [20], [24], and [33] specify dedicated algorithms for multi-aperture
SAR processing. The approach presented in [24] and further elaborated in [30] introduces a phase correction that is applied to the raw data to resample the signal in azimuth, while the technique proposed in [20] introduces an algorithm based on a generalization of the sampling theorem that allows for the unambiguous recovery of the azimuth spectrum from multiple aliased subaperture signals. The method is elaborated in several follow-on papers $[27,28$, $31,32,36$ ]. Finally, [33] and [34] bring up another space-time approach for application in small satellite constellations forming a sparse array. The basic idea is to minimize the overall noise power by a trade-off between a spatial filtering of the azimuth signal to suppress Doppler ambiguities, which corresponds to the method presented in [20], and a matched filter.

Besides, alternative concepts not directly building on conventional SAR are brought up, as a squinted SAR configuration in combination with beamforming [8]. Further, a very general approach based on a multi-satellite constellation forming a sparsely distributed radar sensor is developed and an optimum way of processing in the space-time domain is derived in [12] and [19]. Furthermore, [26] presents the "SAR train" that consists of a multi-satellite constellation which is distributed in along-track direction and uses spread spectrum waveforms for transmission.

The paper is organized as follows. Basic properties of the spatial sampling of multi-aperture SAR systems are summarized in Section II. The DBF algorithm in azimuth introduced in [20] is briefly recalled in Sections IIIA and IIIB. The new contributions of the paper start in Section IIIC with an illustrative interpretation of the multi-aperture processing, followed by a detailed analysis on how signal, ambiguities, and noise are affected by the DBF network in Sections IIID, IIIE, and IIIF, respectively. Then, in Section IV, a system design example is presented that allows for verifying the theory derived in Section III and demonstrates the potential of the multi-aperture reconstruction algorithm with respect to different performance parameters and in comparison with alternative techniques of processing the azimuth signal. In a next step, error sources are identified and innovative strategies are derived in Section VA. Optimization concepts as pattern tapering on transmit (Section VB), prebeamshaping on receive (Section VC), and adapted beamforming networks (Section VD) are presented and their performance is shown. In the case of prebeamshaping on receive and adapted beamforming networks, the theoretical examination of residual errors, ambiguities, and the scaling of the signal-to-noise ratio (SNR) is extended to the class of cascaded beamforming systems. The paper closes with a discussion containing an outlook on further issues like ScanSAR in multi-aperture systems and sparse array systems. 


\section{MULTI-APERTURE SAR SIGNAL IN AZIMUTH}

In the following, this paper focuses on the characteristics and processing of the azimuth signal of a single platform SAR system. In order to provide a better understanding, this section recalls basic relationships of multi-aperture systems regarding spatial sampling in along-track dimension.

A. Effective Phase Center, Virtual Sample Position, and Spatial Sampling in Along-Track

The phase center of an antenna represents the "effective" position of the transmitted or received signal and it is situated in the center of the respective antenna or aperture. As in multi-aperture systems a single transmit antenna is combined with a number of receiving channels, the positions of transmitter (Tx) and receiver $(\mathrm{Rx})$ do in general not coincide. Such systems of spatially separated transmit and receive apertures can be approximated by a virtual system where both coincide midway between the transmitter and respective receiver and a constant phase term is added. This position is called "effective phase center" and represents the spatial position of the received signal. The positions of the effective phase centers in azimuth of a single platform system are shown in Fig. 2 on the top. In this case the spacing of the samples is equal to half of the receive aperture spacing $\Delta x$. If we consider now the spatial sample positions for a train of pulses transmitted with PRF, we obtain the sampling scenario as shown in Fig. 2 on the bottom. For every transmitted pulse, $N$ signals are received whose positions in azimuth are determined by the positions of the receivers with respect to the transmitting aperture. Hence, the gaps between samples received for a single pulse are defined by the distance between the receiving channels, while the distance between two subsequent pulses is determined by $v_{s} / \mathrm{PRF}$. Below, the relation between spatial sampling and the system parameters as aperture position, sensor velocity $v_{s}$, and PRF is derived and the most interesting cases are presented.

\section{B. Uniform Sampling}

In the optimum case all gathered samples are distributed uniformly along the synthetic aperture. To obtain these equally spaced samples in a system of $N$ sensors moving with $v_{s}$, the following relation between PRF and the along-track displacements $x_{j}$ of the receive apertures $j=\{2, \ldots, N\}$ relative to the position $x_{1}$ of receiver 1 have to be fulfilled:

$$
x_{j}-x_{1}=\frac{2 \cdot v_{s}}{\operatorname{PRF}}\left(\frac{j-1}{N}+k_{j}\right), \quad k_{j} \in Z .
$$

In a single platform system with an array antenna, the along-track displacement between adjacent
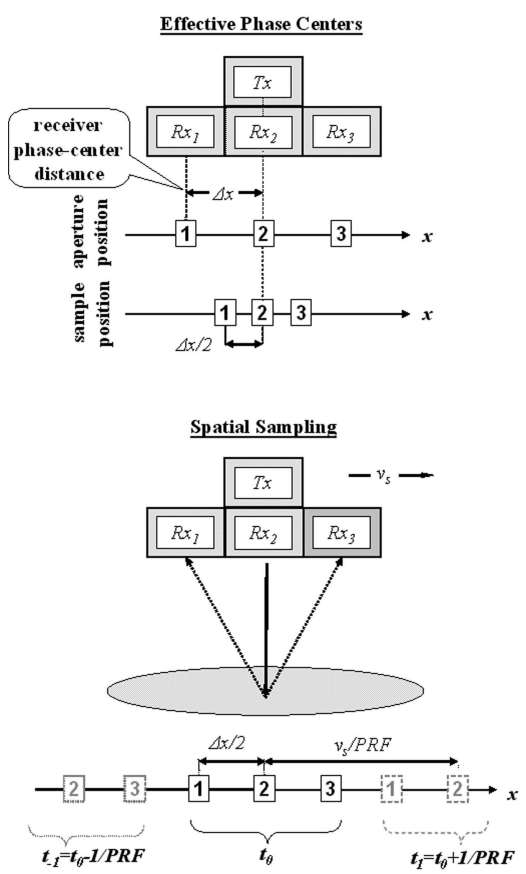

Fig. 2. Aperture position and corresponding effective phase center giving spatial position of sample in azimuth direction $x$ (top) and resulting spatial sampling (bottom) for three subsequent pulses transmitted at $t_{-1}$ (dotted), $t_{0}$ (solid), and $t_{1}$ (dashed). Sample spacing for single transmit pulse is determined by aperture distance $\Delta x$ while offset between samples of subsequent pulses depends on sensor velocity $v_{s}$ and PRF.

subapertures is constant and denoted by $\Delta x$ and all $k_{j}$ of (4) become zero, resulting in expression (5) giving the optimum PRF

$$
\mathrm{PRF}=\frac{2 \cdot v_{s}}{N \cdot \Delta x} .
$$

The optimum value is referred to as "uniform" PRF as it fulfills the timing requirement for uniformly distributed samples and yields a data array equivalent to that of a single-aperture ("monostatic") system operated with $N \cdot$ PRF. Any deviation from the requirements imposed by (4) and (5) will result in nonequally spaced samples along the synthetic aperture ("nonuniform sampling") and the gathered data array does no longer correspond to a monostatic signal and cannot be processed by conventional monostatic algorithms without performance degradation. Hence, classic multi-aperture systems with split antenna in azimuth dimension underlie stringent operational constraints or require appropriate preprocessing to correct for perturbations caused by nonuniformly spaced samples (cf. Section III).

\section{Special Sampling Scenarios}

To complete this section, two special sampling scenarios are presented. Firstly, certain PRFs result in an interleave of samples originating from different transmit pulses in a way such that again all samples 

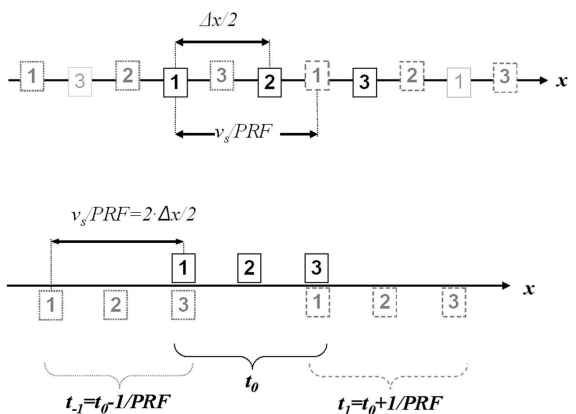

Fig. 3. Spatial sampling in azimuth direction $x$ originating from three subsequent transmit pulses separated by $\Delta t=\mathrm{PRF}^{-1}$ and emitted at $t_{-1}$ (dotted), $t_{0}$ (solid), and $t_{1}$ (dashed) yielding interleaved uniform sampling (top) and spatially coinciding samples of channels 1 and 3 (bottom).

are spaced equally, yielding a uniform sampling of higher order (cf. Fig. 3, top). This means that only after $k$ pulses (5) is fulfilled and no samples coincide spatially, yielding further possible PRFs of uniform sampling, $\mathrm{PRF}_{\text {uni, },}$, given by (6). The inequality for $k$ excludes those values, where the timing requirement is fulfilled, but spatial samples coincide

$$
\begin{aligned}
\mathrm{PRF}_{\text {uni, }, k} & =k \cdot \frac{2 \cdot v_{s}}{N \cdot \Delta x}=k \cdot \mathrm{PRF}_{\text {uni }} \\
k & \neq \frac{p \cdot N}{m}, \quad m \in\{1, \ldots, N-1\}, \quad p, k \in \mathbb{N} .
\end{aligned}
$$

A second interesting case arises when samples of different transmit pulses received by apertures $j$ and $i$ coincide spatially as sketched in Fig. 3, bottom. This condition is defined generally in (7) and simplified for a single platform system in (8), yielding the respective $\mathrm{PRF}_{c}$ specified by $m$ and $n$. Note that the sampling becomes uniform if one of the respective coinciding channels is switched off

$$
\begin{aligned}
\frac{\Delta x_{i}}{2}+k \cdot \frac{v_{s}}{\mathrm{PRF}_{c}} & =\frac{\Delta x_{j}}{2}+l \cdot \frac{v_{s}}{\mathrm{PRF}_{c}}, \quad k, l \in \mathbb{N} \\
\mathrm{PRF}_{c, n, m} & =\frac{2 \cdot v_{s}}{\Delta x} \cdot \frac{n}{m}=\frac{\mathrm{PRF}_{\mathrm{uni}}}{N} \cdot \frac{n}{m}, \\
m & \in\{1, \ldots, N-1\}, \quad n \in \mathbb{N} \wedge n<m \cdot N .
\end{aligned}
$$

\section{DIGITAL BEAMFORMING ON RECEIVE: MULTI-APERTURE RECONSTRUCTION ALGORITHM}

Most of the suggested systems propose to simply operate a multi-aperture antenna in azimuth by interleaving the samples of the different receiving channels without further specific processing steps $[10,11,14,18]$. Consequently, the timing requirement of (5) has to be fulfilled to obtain a signal that is equivalent to a monostatic signal, while in any other case the sample positions deviate from the ideal positions, but are treated as if the signal was sampled uniformly which leads to a degraded system performance as discussed later. In this case, a further processing of the signal is required before conventional monostatic algorithms can be applied to focus the signal. In this section, a technique called Multi-Aperture Reconstruction Algorithm that is suited to process multi-aperture signals is presented, investigated, and compared with alternative approaches. This algorithm was first proposed in [20], and it is based on solving a system of linear equations to unambiguously recover the formerly aliased azimuth spectrum even in case of a nonoptimum PRF, i.e., nonuniformly spaced data samples. Although in the following focus is turned to single platform systems, the algorithm has great potential for any multi-aperture system, be it a distributed SAR with multiple satellites or single antenna systems like the high-resolution wide-swath (HRWS) SAR [14] or the dual receive antenna mode of TerraSAR-X [25].

\section{A. Multi-Aperture Impulse Response and Quadratic Approximation}

In a multi-aperture configuration consisting of a receiver $j$ separated by $\Delta x_{j}$ from the transmitter, the azimuth response $h_{s, j}(t)$ for a point-like target at azimuth time $t=0$, slant range $R_{0}$ and for a carrier wavelength $\lambda$ is described by (9), for the time neglecting the azimuth envelope of the signal. The phase is determined by the lengths of transmit and respective receive path which are approximated by the square roots assuming a straight sensor trajectory

$$
\begin{aligned}
& h_{s, j}\left(t, \Delta x_{j}\right) \\
& \quad=\exp \left[-j \frac{2 \pi}{\lambda}\left(\sqrt{R_{0}^{2}+\left(v_{s} \cdot t\right)^{2}}+\sqrt{R_{0}^{2}+\left(v_{s} \cdot t-\Delta x_{j}\right)^{2}}\right)\right] .
\end{aligned}
$$

Quadratic approximation of (9) yields (10) that allows for relating the multi-aperture point target response to the monostatic response $h_{s}(t)$ by a constant phase shift $\Delta \varphi_{j}=-\pi \cdot \Delta x_{j}^{2} /\left(2 \lambda \cdot R_{0}\right)$ and a time delay $\Delta t_{j}=\Delta x_{j} /\left(2 v_{s}\right)$, what corresponds to the effective phase center position midway between transmitter and receiver as introduced in Section IIA. In Doppler frequency (' $f$ ') domain, this relation between a monostatic impulse response $H_{s}(f)$ and the multi-aperture response of channel $j, H_{s, j}(f)$, is described by the filter $H_{j}(f)$ (cf. (11) and (12))

$$
\begin{aligned}
h_{s, j}(t) \cong & \exp \left[-j \frac{4 \pi}{\lambda} R_{0}\right] \cdot \exp \left[-j \frac{\pi \cdot \Delta x_{j}^{2}}{2 \lambda \cdot R_{0}}\right] \\
& \cdot \exp \left[-j \frac{2 \pi \cdot v_{s}^{2}}{\lambda} \frac{\left(t-\left(\Delta x_{j} / 2 v_{s}\right)\right)^{2}}{R_{0}}\right] \\
= & h_{s}\left(t-\Delta t_{j}\right) \cdot e^{j \Delta \varphi_{j}}
\end{aligned}
$$




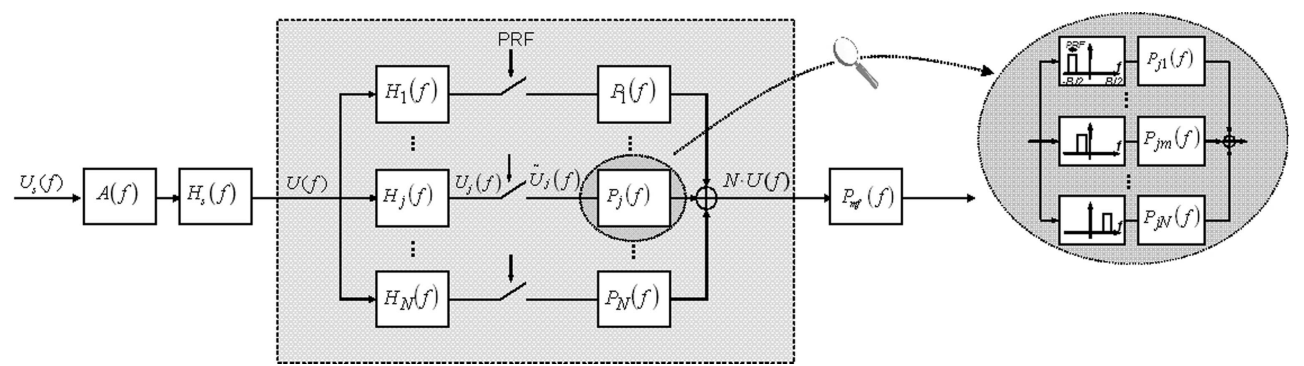

Fig. 4. Left: Block diagram of multi-channel SAR system with "reconstruction" of $N$ subsampled channels by filters $P_{j}(f)$. Right (zoom): Each $P_{j}(f)$ consists of $N$ bandpass filters $P_{j m}(f)$ valid on a sub-band of width PRF.

$$
\begin{aligned}
H_{s, j}(f) & =H_{j}(f) \cdot H_{s}(f) \\
H_{j}(f) & =\exp \left[-j \frac{\pi \cdot \Delta x_{j}^{2}}{2 \lambda \cdot R_{0}}\right] \cdot \exp \left[-j \frac{\pi \cdot \Delta x_{j}}{v_{s}} \cdot f\right] .
\end{aligned}
$$

The multi-aperture response can hence be separated into the influence of the multi-aperture system described by $H_{j}(f)$ and the conventional SAR impulse response given by $H_{s}(f)$. Further, for single platform systems the same azimuth antenna pattern $a(t)$ and $A(f)$, respectively, can be assumed for all channels. This yields the block diagram of Fig. 4, where $u_{s}(t)$ and $U_{s}(f)$, respectively, describe the scene reflectivity. Filtering of the scene with $A(f) \cdot H_{s}(f)$ yields the monostatic SAR signal in Doppler domain, $U(f)$, with its time domain representation $u(t)$. The multi-aperture SAR signal of channel $j$ is then given by $U_{j}(f)$ and $u_{j}(t)$, that are related to $U(f)$ and $u(t)$ by $H_{j}(f)$ according to (13) and its time domain representation $h_{j}(t)$, respectively

$$
U_{j}(f)=H_{j}(f) \cdot U(f) .
$$

\section{B. Theoretical Background}

The multi-aperture reconstruction algorithm is founded on a generalization of the sampling theorem according to which $N$ independent representations of a signal, each subsampled at $1 / N$ th of the signal's Nyquist frequency, allow for the unambiguous "reconstruction" of the original signal from the aliased Doppler spectra of the $N$ representations. This means that a band-limited signal $U(f)$ is uniquely determined in terms of the responses $H_{j}(f)$ of $N$ linear systems with input $U(f)$, sampled at $1 / N$ th of the Nyquist frequency. The functions $H_{j}(f)$ may be chosen in a quite general way, but not arbitrarily [38, 39]. Transferred to a multi-aperture SAR system, $U(f)$ gives the monostatic SAR signal while the functions $H_{j}(f)$ represent the 'channel' between the transmitter and each receiver $j$ with respect to the monostatic impulse response (cf. Fig. 4 and Section A). Note that in principle the complete multi-channel SAR signal model of (9) including the two-way patterns of the respective channels can be used for a complete reconstruction of the scene reflectivity, but for reasons of simplicity only the quadratically approximated system model of $\mathrm{A}$ is considered in the following.

The received signals are sampled in azimuth by PRF and hence the maximum signal bandwidth is $N \cdot$ PRF. A compact characterization of the whole system is then given by the matrix $\mathbf{H}(f)$, that contains all channel representations $H_{j}(f)$ shifted by integer multiples of the PRF according to (14)

$$
\mathbf{H}(f)=\left[\begin{array}{ccc}
H_{1}(f) & \cdots & H_{N}(f) \\
H_{1}(f+\mathrm{PRF}) & \cdots & H_{N}(f+\mathrm{PRF}) \\
\vdots & \ddots & \vdots \\
H_{1}(f+(N-1) \cdot \mathrm{PRF}) & \cdots & H_{N}(f+(N-1) \cdot \mathrm{PRF})
\end{array}\right] .
$$

Then, as shown in [39], the inversion of $\mathbf{H}(f)$ yields a matrix $\mathbf{P}(f)$ that contains in its rows $N$ functions $P_{j}(f)$ that are decomposed by the columns in $N$ functions $P_{j m}(f)$ defined on sub-bands $m$ of width PRF that make up the Doppler spectrum (cf. Fig. 4 and (15)). As we will see later, the scaling by $N$ is reasonable from an energy point of view. Note that in the considered case $\mathbf{H}(f)$ is invertible as long as samples of different receive apertures do not coincide spatially

$$
\begin{aligned}
\mathbf{P}(f) & =N \cdot \mathbf{H}^{-1}(f) \\
& =\left[\begin{array}{cccc}
P_{11}(f) & P_{12}(f+\mathrm{PRF}) & \cdots & P_{1 N}(f+(N-1) \mathrm{PRF}) \\
P_{21}(f) & P_{22}(f+\mathrm{PRF}) & \cdots & P_{2 N}(f+(N-1) \mathrm{PRF}) \\
\vdots & \vdots & \ddots & \vdots \\
P_{N 1}(f) & P_{N 2}(f+\mathrm{PRF}) & \cdots & P_{N N}(f+(N-1) \mathrm{PRF})
\end{array}\right] .
\end{aligned}
$$

The aliased frequencies in the Doppler spectra of the individual channels are then suppressed and consequently the original signal $U(f)$ is recovered by filtering each of the multi-aperture channels $j$ with its appropriate "reconstruction" filter $P_{j}(f)$ and subsequent coherent combination of all weighted receiver channels (cf. Fig. 4). To complete Fig. 4, the conventional monostatic SAR focusing filter $P_{m f}(f)$ is included. 


\section{Illustration of Principle}

In this section, an illustrative approach to explain the principle and limitations of the multi-aperture reconstruction algorithm is given. Consider a system according to Fig. 4 with exemplary $N=2$ apertures and $U(f)$ describing a monostatic SAR signal while $U_{j}(f)=H_{j}(f) \cdot U(f)$ represents the signal at receiver $j$. At first, assume $U(f)$ spectrally limited to [-PRF,PRF]. Then, Fig. 5 on the left shows the spectrum of the band-limited signal "seen" at the receiver $j$ before sampling (top) and its periodic continuation after sampling with PRF (middle) yielding $\tilde{U}_{j}(f)$, given in (16). For convenience we introduce the notation $U_{j k}(f)=U_{j}(f+k \cdot \mathrm{PRF})$ where the index $k$ indicates a shift by $k \cdot$ PRF in Doppler domain

$$
\begin{aligned}
\tilde{U}_{j}(f) & =\sum_{k=-\infty}^{\infty} U_{j}(f+k \cdot \mathrm{PRF})=\sum_{k=-\infty}^{\infty} U_{j k}(f) \\
& =\sum_{k=-\infty}^{\infty} U(f+k \cdot \mathrm{PRF}) \cdot H_{j}(f+k \cdot \mathrm{PRF}) .
\end{aligned}
$$

As can be observed, for any frequency, the subsampled and aliased signal consists of not more than two (in general $N$ ) shifted and superimposed spectra, as not more than one spectrum (in general $N-1$ spectra) of the periodic continuation overlaps with the original spectrum. Hence, the spectra $\tilde{U}_{1}(f)$ and $\tilde{U}_{2}(f)$ can be weighted and combined in such a way, that the component of the original spectrum is recovered (cf. (17), top), while the back-folded component is cancelled (cf. (17), bottom). With $P_{j m}(f)$ denoting the reconstruction filter for receiver $j$ on the Doppler frequency interval $m$ (cf. Fig. 4), this requires the following equations to hold in the interval $I_{1}$ (cf. Fig. 5, middle, left):

$$
\begin{aligned}
& P_{11}(f) \cdot H_{1}(f) \cdot U(f)+P_{21}(f) \cdot H_{2}(f) \cdot U(f) \\
& \quad \stackrel{!}{=} N \cdot U(f) \\
& P_{11}(f) \cdot H_{1}(f+\mathrm{PRF}) \cdot U(f+\mathrm{PRF})+P_{21}(f) \\
& \quad \cdot H_{2}(f+\mathrm{PRF}) \cdot U(f+\mathrm{PRF}) \\
& \quad \stackrel{!}{=} 0 .
\end{aligned}
$$

For a uniform sampling, no processing needs to be applied, i.e., the samples of all signals have to be simply interleaved and hence it seems reasonable to set the arbitrary normalization factor equal to $N$, as this ensures a magnitude equal to 1 of the filters $P_{j m}$ in this special case. Setting up the corresponding equations on the interval $I_{2}$ and shifting them to $I_{1}$ yields (18) which allows for setting up the linear

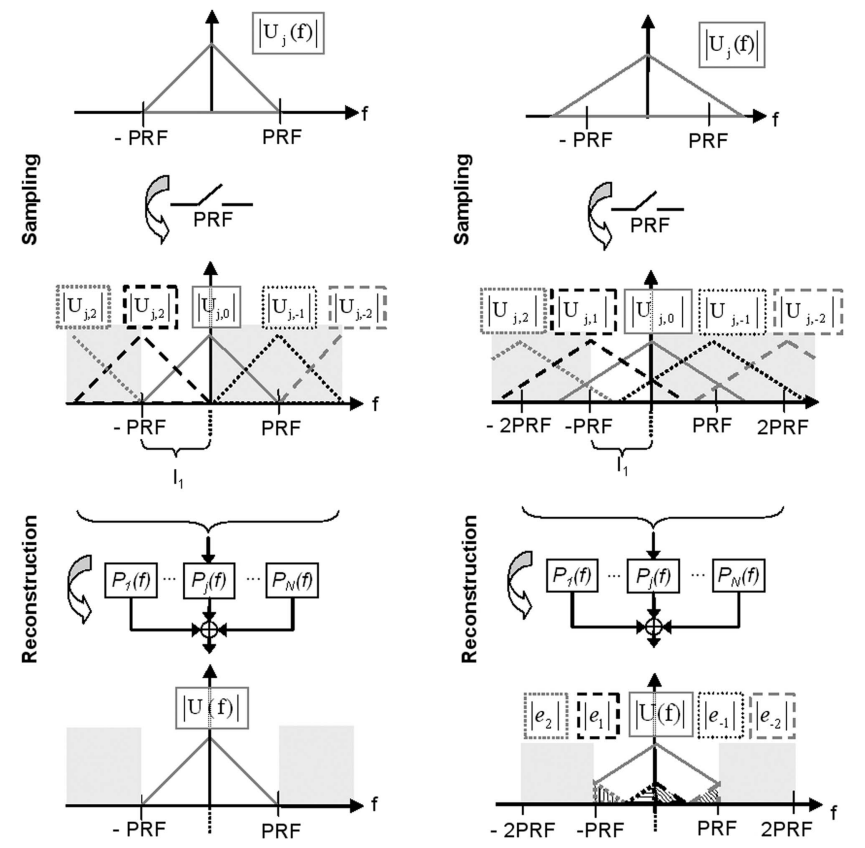

Fig. 5. Signal spectrum at receiver $j$ before (top) and after (middle) sampling with PRF and after reconstruction (bottom) taking into account all receivers. Left shows signal band-limited to [-PRF,PRF] that is correctly recovered while the bandwidth on the right exceeds $[-\mathrm{PRF}, \mathrm{PRF}]$ entailing erroneous contributions $e_{k}$.

system of (19) that corresponds to (15)

$$
\begin{aligned}
& \begin{aligned}
P_{12}(f & +\mathrm{PRF}) \cdot H_{1}(f+\mathrm{PRF}) \cdot U(f+\mathrm{PRF}) \\
& +P_{22}(f+\mathrm{PRF}) \cdot H_{2}(f+\mathrm{PRF}) \cdot U(f+\mathrm{PRF}) \\
& \stackrel{!}{=} N \cdot U(f+\mathrm{PRF})
\end{aligned} \\
& \begin{aligned}
P_{12}(f+\mathrm{PRF}) \cdot H_{1}(f) \cdot U(f)+P_{22}(f+\mathrm{PRF}) \cdot H_{2}(f) \cdot U(f) \\
\quad \stackrel{!}{=} 0
\end{aligned} \\
& \mathbf{H}(f) \cdot \mathbf{P}(f)=N \cdot 1 \Leftrightarrow \mathbf{P}(f)=N \cdot \mathbf{H}^{-1}(f) .
\end{aligned}
$$

In a next step, we analyze a scenario where the bandwidth of the signal exceeds $N$. PRF. For $N=2$, an example for the spectrum of such a signal is given in Fig. 5 on the right, before (top) and after (middle) sampling. In contrast to the band-limited case, the sampled signal $\tilde{U}_{j}(f)$ consists of up to three contributions, as the spectra of the periodic continuation may overlap. For the general case, this means that more than $N$ spectra may coincide at a certain Doppler frequency. From the mathematical point of view this results in a linear system of equations that is underdetermined and consequently the original spectrum can in general not be reconstructed exactly. As shown in [28], a complete suppression of the contributions from the shifted spectra is not achieved, because the filters $P_{j}(f)$ are determined as if the signal bandwidth was limited to $\pm N \cdot \mathrm{PRF} / 2$. Hence only the ambiguous 
energy within the band $[-N \cdot \mathrm{PRF} / 2, N \cdot \mathrm{PRF} / 2]$ of the original signal is cancelled by the reconstruction. All energy outside this band is not well suppressed and finally gives rise to ambiguous contributions $e_{k}$ in the reconstructed signal (cf. Fig. 5 bottom right) that is specified in the following section. How the ambiguous energy can be reduced, e.g. by selecting appropriate sub-bands or weighting of the azimuth spectrum during the reconstruction is discussed in Section V.

\section{Signal Power}

In the following, the signal power is defined as the mean energy of the unambiguous signal, which is limited by the system bandwidth in azimuth $I_{s}=N \cdot \mathrm{PRF}$ and eventually further confined by the processed Doppler bandwidth $B_{D} \leq I_{s}$. To determine the signal power, at first the output signal power of the reconstruction network is determined. Remember that the filters $P_{j}(f)$ are chosen such that the "original" signal received by a single aperture $U(f)$ is reconstructed. Recall furthermore that the spectral weighting of $U(f)$ depends only on its envelope defined by the joint pattern $A(f)$ of the transmitter and a single receiver element. This means that we basically obtain a signal that is not dependent on the reconstruction filter network up to a constant scaling factor of $N$ as given in (17). This factor accounts for a digital summation yielding an amplitude gain increased by a factor of $N$ with respect to a single channel and consequently the power is amplified by $N^{2}$ compared with the input signal power $p_{s, e l}$. Then, the signal power $p_{s}$ is determined by the mean squared value of $N \cdot U(f)$ limited to the system bandwidth in azimuth $I_{S}=[-N \cdot \mathrm{PRF} / 2, N \cdot \mathrm{PRF} / 2]$ (cf. (20)). The limitation to $I_{S}$ is expressed by the rectangular window function $\operatorname{rect}\left(f / I_{s}\right)$ and the calculation of the mean value within this interval is indicated by the operator $\mathbf{E}[]^{1}$

$$
p_{s}=N^{2} \cdot \mathbf{E}\left[\left|U(f) \cdot \operatorname{rect}\left(f / I_{S}\right)\right|^{2}\right]=N^{2} \cdot p_{s, e l} .
$$

If the signal is focused with a defined bandwidth $B_{D}$ only this part of $I_{S}$ is used for the compression, what can be understood as an additional lowpass filter of width $B_{D}$ that is applied to the signal. This filtering is equivalent to a fixed integration time resulting in an azimuth spectrum of width $B_{D}$. For the case where the system is operated with a constant duty-cycle, the average transmit power per time is constant. This means that the resulting energy after focusing is independent from the PRF, as e.g. the decreasing

\footnotetext{
${ }^{1}$ Note that for deterministic signals as $U(f)$ the operator $\mathbf{E}[$.] is identical to an integration over frequency normalized by the interval width, while for stochastic processes $\mathbf{E}[$.] describes the expectation value. As both give a measure of the power, in the following the same operator symbol is used to provide a consistent notation.
}

energy per sample for a higher PRF is compensated by the fact that more samples are gathered within the integration time and then combined during azimuth compression. We obtain (21), giving $p_{S, B D}$ that describes the signal power after focusing with $B_{D}$, where the mean value is calculated on the original interval $I_{s}$ and a "white" scene is assumed which means that $|U(f)|$ can be described by the signal envelope $A(f)$

$$
\begin{aligned}
p_{s, B_{D}} & =N^{2} \cdot \mathbf{E}\left[\left|U(f) \cdot \operatorname{rect}\left(f / B_{D}\right)\right|^{2}\right] \\
& =N^{2} \cdot \mathbf{E}\left[\left(A(f) \cdot \operatorname{rect}\left(f / B_{D}\right)\right)^{2}\right] .
\end{aligned}
$$

E. Residual Reconstruction Error, Error Power, and Azimuth Ambiguities

Section $\mathrm{C}$ illustrated that energy outside the band $[-N \cdot \mathrm{PRF} / 2, N \cdot \mathrm{PRF} / 2]$ of the original signal is not cancelled by the algorithm and disturbs the unambiguous reconstruction of the multi-aperture SAR signal. With knowledge of the overall configuration and the antenna patterns, this section derives the spectral "error" that remains from these aliased parts after the system of reconstruction filters. The further development considers the sampled signal at receiver $j$ in (16) and focuses on the $k$ th continuation of the original spectrum $U_{j}(f+k \cdot \mathrm{PRF})=$ $U_{j k}(f)$. It is now of interest, how the ambiguous parts of $U(f)$, i.e., the contributions outside the original frequency band $[-N \cdot \mathrm{PRF} / 2, N \cdot \mathrm{PRF} / 2]$, are folded back by the sampling. As the further processing of $U_{j k}(f)$ by the filters $P_{j m}(f)$ is defined on sub-bands $m$ of width PRF, the original band is split into respective intervals $I_{m}=[(-N / 2+m-1)$. PRF, $(-N / 2+m) \cdot$ PRF], where $m=1, \ldots, N$. The ambiguous contributions of $U_{j k}(f)$ are located at frequencies that deviate more than $\pm N \cdot \mathrm{PRF} / 2$ from the center frequency $-k \cdot \mathrm{PRF}$, i.e., $|f+k \cdot \mathrm{PRF}|$ $>N / 2 \cdot$ PRF. Further, only contributions within $[-N \cdot \mathrm{PRF} / 2, N \cdot \mathrm{PRF} / 2]$ are of relevance, yielding the following expressions for the bands where the ambiguous parts are located:

$$
\begin{array}{r}
\frac{N}{2} \mathrm{PRF}-k \cdot \mathrm{PRF} \leq f \leq \frac{N}{2} \mathrm{PRF}, \quad k>0 \\
-\frac{N}{2} \mathrm{PRF} \leq f \leq-\frac{N}{2} \mathrm{PRF}-k \cdot \mathrm{PRF}, \\
k<0 .
\end{array}
$$

Consequently, depending on $k$, certain sub-bands of the spectrum are not cancelled by the reconstruction algorithm and have to be considered when determining the error. Assuming a symmetrical pattern, i.e., $A(f)=A(-f)$, the problem is symmetrical and it is sufficient to concentrate on $k>0$ and (22). In this case, sub-bands $I_{m}$ up from index $m_{0}=\max \{N-k+1,1\}$ contribute to the error. Note that choosing $m_{0}=1$ 
does not lead to wrong results as the respective summands are zero due to the inverse filtering by $P_{j m}(f)$. In a next step the processing of the signal is taken into account by considering the weighting of the signal $U_{j k}(f)$ with the filters $P_{j m}(f)$ over all sub-bands $I_{m}$ where the signal is not properly reconstructed. Then we sum over contributions from all receiver channels $j$. This yields $e_{k}(f)$ that describes the spectrum of the remaining output error due to aliasing of the input signal caused by the $k$ th backfolded spectrum (cf. (24) and Fig. 5 bottom right). $U_{j}(f)$ is expressed by the monostatic SAR signal $U(f)$ multiplied by the respective $H_{j}(f)$. Again, index $k$ indicates a shift from $f$ to $f+k$. PRF due to the sampling and the signal is confined to the sub-band $I_{m}$ by a rectangular window of width PRF around a center frequency $f_{0, m}=(-N / 2+m-1 / 2) \cdot \mathrm{PRF}$ represented by $\operatorname{rect}\left(\left(f-f_{0, m}\right) / \mathrm{PRF}\right)$

$$
\begin{aligned}
e_{k}(f) & =\sum_{m=m_{0}}^{N} \sum_{j=1}^{N} \underbrace{U_{k}(f) \cdot H_{j k}(f)}_{U_{j k}(f)} \cdot \underbrace{P_{j}(f) \cdot \operatorname{rect}\left(\frac{f-f_{0, m}}{\mathrm{PRF}}\right)}_{P_{j m}(f)} \\
& =U_{k}(f) \cdot \sum_{m=m_{0}}^{N} \sum_{j=1}^{N} H_{j k}(f) \cdot P_{j m}(f) .
\end{aligned}
$$

The complete ambiguous spectrum due to aliasing is obtained by summation of all individual ambiguous contributions after reconstruction, $e_{k}$, yielding $e_{\Sigma}(f)$ in (25), where the factor 2 accounts for both signs of $k$

$$
\begin{aligned}
e_{\Sigma}(f) & =2 \cdot \sum_{k=1}^{\infty} e_{k}(f) \\
& =2 \cdot \sum_{k=1}^{\infty}\left(U_{k}(f) \cdot \sum_{m=m_{0}}^{N} \sum_{j=1}^{N} H_{j k}(f) \cdot P_{j m}(f)\right) .
\end{aligned}
$$

Expression (25) can be divided into the characteristics of the azimuth signal and the overall configuration. The signal spectrum $U_{k}(f)$ is defined by size and tapering of the transmit antenna and the single receive aperture, while the second part of (25) is defined by the products $H_{j k}(f) \cdot P_{j m}(f)$ that depend on the relative positions of all apertures. Relating the ambiguous power given by the mean squared amplitude of $e_{\Sigma}(f)$ with the signal power of (20) results in an equivalent azimuth ambiguity-to-signal ratio for multi-aperture SAR systems $\left(\mathrm{AASR}_{N}\right)$ that takes into account the weighting and coherent combination of the individual channels by the beamforming network

$$
\operatorname{AASR}_{N}=\frac{\mathbf{E}\left[\left|e_{\Sigma}(f)\right|^{2}\right]}{p_{s}} .
$$

So far the $\mathrm{AASR}_{N}$ was calculated for the complete Doppler bandwidth given by $N$. PRF. In a next step, the respective processed bandwidth and the associated lowpass filtering are included, yielding the $\mathrm{AASR}_{N, B D}$ for the image. Therefore $e_{\Sigma}(f)$ is band-limited to $B_{D}$ by a rectangular window $\operatorname{rect}\left(f / B_{D}\right)$ before the ambiguous power is calculated

$$
\operatorname{AASR}_{N, B_{D}}=\frac{\mathbf{E}\left[\left|e_{\Sigma}(f) \cdot \operatorname{rect}\left(f / B_{D}\right)\right|^{2}\right]}{p_{s, B_{D}}} .
$$

In order to obtain the response of the residual ambiguity of order $k$ in the image, the respective ambiguous contribution $e_{k}$ defined by (24) has to be focused by the used SAR processor.

\section{F. SNR Scaling in Digital Beamforming Networks $-\Phi_{b f}$}

In this section, the influence of the DBF network on signal and noise power is investigated and a parameter that characterizes the impact of a network of digital filters on the SNR is derived. As under certain conditions the SNR is improved by the processing, we abandon the term "noise figure" but talk about "SNR scaling" in beamforming networks and denote it by $\Phi_{b f}$ to avoid confusion with existing terminology.

Consider the multi-aperture system as a linear system of $N$ channels $j$ with an input signal $u_{j}(t)$ and additive white Gaussian receiver noise components $n_{j, B}(t)$ that are mutually uncorrelated between the channels. The corresponding spectra are given by $U_{j}(f)$ and $n_{j, B}(f)$ and the signal-to-noise ratio is denominated by $\mathrm{SNR}_{e l, j}$. The system model of a single channel $j$ is shown in Fig. 6. After reception, the signal is amplified in the low-noise amplifier (LNA) that is characterized by its power gain $G_{j}$, amplitude gain $g_{j}$, and its noise figure with respect to power $\left(F_{j}\right)$ and amplitude $\left(f_{j}\right)$. Under the realistic assumption of a sufficiently high $G_{j}$ the $n_{j, B}(f)$ are the dominating sources of thermal noise and hence all other thermal noise sources afterwards are neglected. After amplification the signal is sampled in azimuth with a ratio of PRF yielding a subsampled and consequently aliased time-discrete signal. The quantization error induced by the sampling is modelled as an additive noise source $n_{q j}$ and assumed to be uniformly distributed and spectrally white as proposed in [40]. Further, we assume the granular error dominant and consequently no SNR loss caused by clipping is encountered. The signal of each channel $j$ is then digitally filtered by a Doppler frequency dependent filter $P_{j}(f)$ that is defined on a bandwidth of $N \cdot$ PRF. Finally, the outputs $u_{\text {out }, j}[k]$ of all $N$ branches are combined coherently yielding the output signal $u_{\text {out }}[k]$ (cf. Fig. 6). As the system is linear and only additive noise sources are considered, signal and noise power can be analyzed separately, when investigating the influence of the reconstruction network on the SNR. Note that in the following, "power" is used to describe 


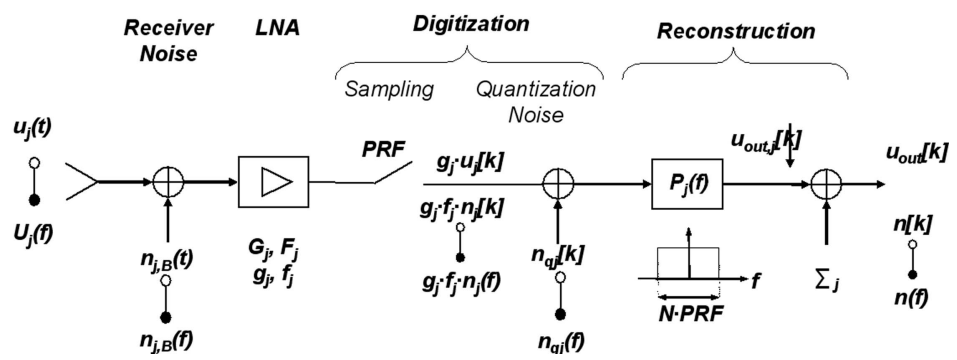

Fig. 6. System model for single channel of multi-aperture system. After reception of $U_{j}(f)$, receiver noise is added. Then RF signal is amplified and digitized before filtering and coherent combination of all channels.

the average energy per time or frequency, respectively. The normalisation to the respective interval width is included in the mathematical operator $\mathbf{E}[$.$] that$ represents the mean value for deterministic signals and the expectation value for stochastic processes. For reasons of simplicity, considerations are carried out in frequency domain and after sampling in Doppler frequency domain, both designated by $f$. After sampling, only Doppler frequency is considered, as reconstruction by the filters $P_{j}(f)$ is carried out in Doppler domain.

First, consider how the beamforming network affects the noise power. In channel $j$, the thermal noise contribution after sampling is $g_{j} \cdot f_{j} \cdot n_{j}(f)$ while $n_{q j}(f)$ accounts for the quantization noise. After digitization, filtering with $P_{j}(f)$, and coherent summation of all channels $j$, (28) describes the overall noise $n(f)$ in the reconstructed data. The output noise power $p_{n}$ is then defined by the mean square value of $n(f)$ given by expression (29)

$$
\begin{aligned}
n(f) & =\sum_{j=1}^{N} P_{j}(f) \cdot\left(n_{j}(f) \cdot g_{j} \cdot f_{j}+n_{q j}(f)\right) \\
p_{n} & =\mathbf{E}\left[|n(f)|^{2}\right] \\
& =\mathbf{E}\left[\left|\sum_{j=1}^{N} P_{j}(f) \cdot\left(n_{j}(f) \cdot g_{j} \cdot f_{j}+n_{q j}(f)\right)\right|^{2}\right] .
\end{aligned}
$$

As $n_{q j}$ and $n_{j}$ are uncorrelated [40], the overall noise power $p_{n}$ simplifies to the sum of the noise powers induced by thermal receiver noise components $p_{n, r x, j}$ and the quantization $\left(p_{n, q}\right)$. Assuming mutually uncorrelated $n_{j}$, the squared sum representing the receiver noise power in (29) simplifies to the sum of squared values (cf. (30))

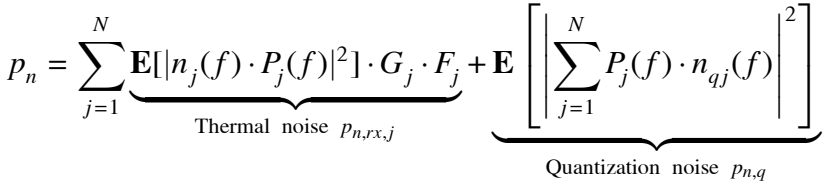

$$
\begin{aligned}
& =\sum_{j=1}^{N} p_{n, r x, j}+p_{n, q} .
\end{aligned}
$$

The power of $n_{j}(f)$ can be expressed by the power at the point of reception, $p_{n, e l, j}$, i.e., the power of $n_{j, B}(f)$, as the sampling changes only the power spectral density of the noise without affecting its power or spectral appearance

$$
p_{n, e l, j}=\mathbf{E}\left[\left|n_{j, B}(f)\right|^{2}\right]=\mathbf{E}\left[\left|n_{j}(f)\right|^{2}\right] .
$$

Finally, we assume all subaperture elements $j$ to be identical, which means that we expect the same characteristics for $G_{j}, F_{j}, g_{j}, f_{j}$ and thermal noise of same power $p_{n, e l, j}$ for all elements. Further, the assumption of spectrally white receiver noise allows for separating the noise power and its spectral weighting given by the $P_{j}(f)$, which yields the following expression for the system's output noise power $p_{n}$ that consists of $p_{n, r x}$ and $p_{n, q}$

$$
\begin{aligned}
p_{n} & =\underbrace{p_{n, e l} \cdot G \cdot F \cdot \sum_{j=1}^{N} \mathbf{E}\left[\left|P_{j}(f)\right|^{2}\right]}_{\text {Thermal noise } p_{n, r x}}+\underbrace{\mathbf{E}\left[\left|\sum_{j=1}^{N} P_{j}(f) \cdot n_{q j}(f)\right|^{2}\right]}_{\text {Quantization noise } p_{n, q}} \\
& =p_{n, r x}+p_{n, q} .
\end{aligned}
$$

Due to the mutual correlation of the quantization errors in the channels $j$, the scaling of the error by the network can be stronger than the amplification of the thermal noise. Generally, we expect that the number of bits will be chosen such that the quantization noise in the output signal will be negligible compared with the thermal noise, which means that the overall noise can be approximated by $p_{n, r x}$ defined by the input noise power and an amplification factor determined by the reconstruction filter network (cf. (32)).

In a next step, the influence of the reconstruction network on the signal power is investigated. Taking into account the power gain $G$ of the LNA and considering the scaling of the signal power by $N^{2}$ by the beamforming network (cf. Section D), the signal power after reconstruction $p_{s}$ is given by scaled input noise power $p_{s, e l}$ according to (33)

$$
p_{s}=p_{s, e l} \cdot G \cdot N^{2} \text {. }
$$

Combining (32) and (33), the following expression for the scaling of the SNR by the network is obtained 
[31]

$$
\frac{p_{s, e l} / p_{n, e l}}{p_{s} / p_{n}}=\frac{\mathrm{SNR}_{e l}}{\mathrm{SNR}_{\text {out }}}=\frac{F \cdot \sum_{j=1}^{N} \mathbf{E}\left[\left|P_{j}(f)\right|^{2}\right]}{N^{2}} .
$$

To concentrate on the effect of the reconstruction network, we define the SNR scaling factor $\Phi_{b f}$ of the DBF network by (34) normalized to the optimum value of $F / N$ that is achieved for uniform sampling

$$
\Phi_{b f}:=\frac{\mathrm{SNR}_{e l} / \mathrm{SNR}_{\text {out }}}{\left.\left(\mathrm{SNR}_{e l} / \mathrm{SNR}_{\text {out }}\right)\right|_{\mathrm{PRF}_{\text {uni }}}}=\frac{\sum_{j=1}^{N} \mathbf{E}\left[\left|P_{j}(f)\right|^{2}\right]}{N} .
$$

Note that $\Phi_{b f}$ considers only the relation between $\mathrm{SNR}_{e l}$ and $\mathrm{SNR}_{\text {out }}$ at the respective PRF. This includes the gain by oversampling but does not account for a possible variation of the $\mathrm{SNR}_{e l}$ with the $\mathrm{PRF}$ as it is e.g. encountered for a constant duty cycle.

From a matrix theoretical point of view, the scaling of the receiver noise power in the data as quantified by (35), can be expressed by the sum of the eigenvalues $\lambda_{j}(f)$ of the matrix $\mathbf{P}(f) \cdot \mathbf{P}^{\mathrm{H}}(f)$, where $\mathbf{P}^{\mathrm{H}}$ represents the conjugate transpose of $\mathbf{P}$. For a given PRF the sum over all eigenvalues is constant and consequently the SNR scaling factor $\Phi_{b f}$ is not frequency dependent. The equality of the representations of the SNR scaling factor in (35) and (36) is derived using the relation between the trace of a matrix and the sum over its eigenvalues in combination with the Hilbert-Schmidt norm

$$
\Phi_{b f}=\frac{\sum_{j=1}^{N} \lambda_{j}(f)}{N} .
$$

In 2006 Wang and Bao [41] have shown that for the considered system and the approximations proposed in [20] the scaling of the noise by the reconstruction network as given in (34) can be expressed explicitly by harmonic functions as follows in (37), where the sample positions of receivers $p$ and $r$ are described by their respective sample times $t_{p}$ and $t_{r}$ normalised by $1 / \mathrm{PRF}$. Basically the numerator gives the deviation of the samples from the optimum regular spaced positions given by $2 \pi \cdot n / N$, while the denominator takes the mutual distances between the receivers into account

$$
\begin{aligned}
& \sum_{j=1}^{N} \mathbf{E}\left[\left|P_{j}(f)\right|^{2}\right] \\
& \quad=\frac{1}{N} \sum_{p=1}^{N} \frac{\sum_{n=0}^{N-1} \prod_{r=1, r \neq p}^{N}\left(1-\cos \left(2 \pi \cdot n / N-2 \pi \cdot t_{r} \cdot \mathrm{PRF}\right)\right)}{\prod_{r=1, r \neq p}^{N}\left(1-\cos \left(2 \pi \cdot \operatorname{PRF} \cdot\left(t_{p}-t_{r}\right)\right)\right)} .
\end{aligned}
$$

In a further step, we take the focusing of the data into account to describe the noise in the image, $p_{n, B D}$. Based on the assumption of spectrally white receiver noise, the power spectral density of the noise decreases with increasing PRF while the noise power remains constant [42]. Hence, to derive
TABLE I

Mission Performance Requirements

\begin{tabular}{lcc}
\hline \hline Carrier wavelength & $\lambda$ & $0.031 \mathrm{~m}$ \\
Orbit height & $h_{s}$ & $500 \mathrm{~km}-700 \mathrm{~km}$ \\
Coverage (incident angle) & $\theta_{i}$ & $20^{\circ}-55^{\circ}$ \\
$\begin{array}{l}\text { Swath width } \\
\text { Geometric resolution in azimuth \& } \\
\text { range }\end{array}$ & $\delta_{a z}, \delta_{r g}$ & $100 \mathrm{~km}$ \\
$\begin{array}{l}\text { Range ambiguity suppression } \\
\text { (distributed targets) }\end{array}$ & $R A S R$ & $\leq 1 \mathrm{~m}$ \\
$\begin{array}{l}\text { Azimuth ambiguity suppression } \\
\text { (distributed targets) }\end{array}$ & $\mathrm{AASR}_{N}$ & $\leq-21 \mathrm{~dB}$ \\
$\begin{array}{l}\text { Noise equivalent sigma zero } \\
\text { NESZ }\end{array}$ & $\leq 19 \mathrm{~dB}$ \\
\hline
\end{tabular}

an expression for the noise power that remains in the image, the noise power spectral density (defined by noise power and PRF) in combination with a lowpass filter of bandwidth $B_{D}$ has to be considered after the signals are filtered by the $P_{j}(f)$. This yields (38) for the noise power after focusing, which is derived from (32) extended by the rectangular window $\operatorname{rect}\left(f / B_{D}\right)$ limiting the Doppler spectrum

$$
p_{n, B_{D}}=p_{n, e l} \cdot G \cdot F \cdot \sum_{j=1}^{N} \mathbf{E}\left[\left|P_{j}(f)\right|^{2} \cdot \operatorname{rect}\left(f / B_{D}\right)\right] .
$$

The dependency on the PRF cannot be seen explicitly, but it follows implicitly from the restriction of the filters $P_{j}(f)$ to $B_{D}$ and the definition of the $P_{j}(f)$ on a bandwidth of $N$. PRF over which the mean value is calculated. Hence an increasing PRF in combination with a constant $B_{D}$ means a decreasing spectral part-with respect to $N \cdot \mathrm{PRF}$ - that contributes to the noise power. For $B_{D}=N \cdot \mathrm{PRF}$, (38) is equivalent to (34). The SNR scaling factor with respect to the image $\Phi_{b f, B D}$ cannot be given explicitly, as the relation between input and output signal power depends on the shape of $U(f)$ in combination with $B_{D}$ as defined in (21), but of course $\Phi_{b f, B D}$ can be calculated using (21) and (38).

Note that receiver noise and ambiguous power (cf. Section E) are mutually independent, and thus the resulting interfering power in the data and the image can be simply determined by addition of the respective expressions.

\section{SYSTEM DESIGN EXAMPLE}

\section{A. Requirements and Timing}

In the following, an X-band high-resolution wide-swath SAR system is designed with the basic mission performance requirements of Table I and for two different swath definitions. First, a conventional approach is chosen that is suitable to cover the required incident angle range from $20^{\circ}$ to $55^{\circ}$ with swaths of $100 \mathrm{~km}$ and a variable overlap of $10-20 \%$. This case takes into account the interference of the transmit event and the nadir echo. The detailed 

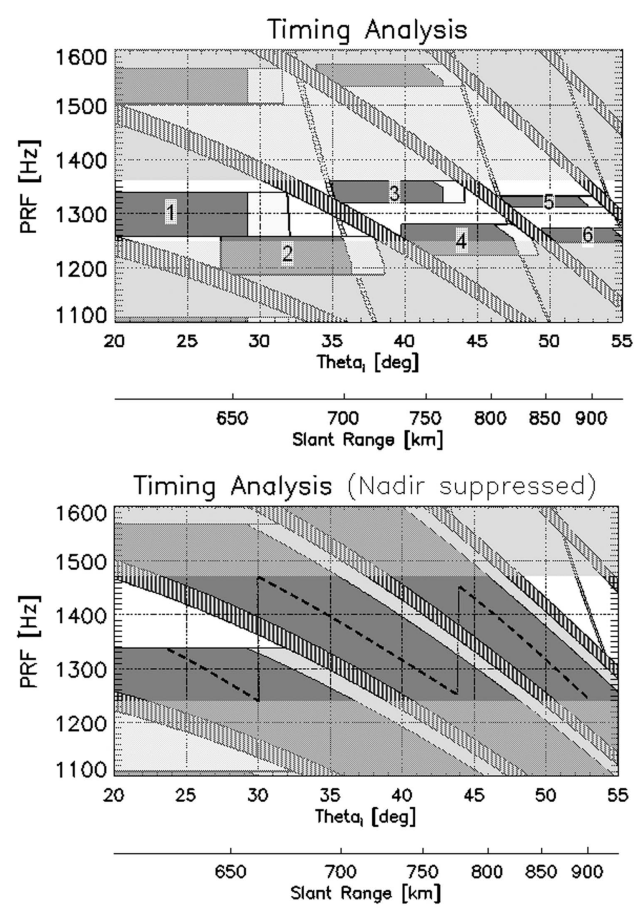

Fig. 7. Top: Timing analysis for orbit height of $580 \mathrm{~km}$ suitable to cover incident angle range from $20-55^{\circ}$ with 6 distinct swaths of width $100 \mathrm{~km}$ taking nadir echo (horizontally streaked) and interference of transmit events (vertically streaked) into account.

Bottom: Timing analysis with nadir echo assumed to be sufficiently suppressed by DBF in elevation. Dashed line indicates center of swath.

relation between swaths and incident angles is given in Table III. Second, a system is regarded, where the beamforming capability in elevation is sufficient for suppressing the nadir echo and consequently the timing depends only on the transmit events. In this case the coverage requirement is modified to enable imaging of a $100 \mathrm{~km}$ swath centered on an arbitrary angle of incidence. A detailed timing analysis with a duty cycle of $15 \%$ yields a suitable orbit height of $580 \mathrm{~km} \pm 10 \mathrm{~km}$, leading to a sensor velocity of $7560 \mathrm{~m} / \mathrm{s}$. Fig. 7 on the bottom shows the result for the suppressed nadir echo, while Fig. 7 on the top accounts for the conventional scenario. In this case the small mutual shift of swaths of the same reference number is due to the variable overlap of adjacent swaths.

\section{B. System Parameters}

The timing shows that for the nominal orbit height a PRF range from $1250 \mathrm{~Hz}$ up to $1350 \mathrm{~Hz}$ is sufficient to ensure the coverage of all six swaths (cf. Fig. 7, top). If the height deviates by $10 \mathrm{~km}$, the minimum PRF decreases to $\sim 1240 \mathrm{~Hz}$. In the case where the nadir is suppressed, the same minimum PRF is chosen. To allow for the imaging of an arbitrarily centered swath, the suitable PRF decreases with increasing incident angle as the dashed
TABLE II

System Parameters

\begin{tabular}{|c|c|c|}
\hline Orbit height & $h_{s}$ & $580 \mathrm{~km} \pm 10 \mathrm{~km}$ \\
\hline Slant Range & $R_{0}$ & $604 \mathrm{~km}-1112 \mathrm{~km}$ \\
\hline Sensor velocity & $v_{s}$ & $7560 \mathrm{~m} / \mathrm{s}$ \\
\hline PRF range & PRF & $1240-1470 \mathrm{~Hz}$ \\
\hline Receive aperture size in azimuth & $d_{a z, r x}$ & $1.6 \mathrm{~m}$ \\
\hline Number of Rx channels in azimuth & $N$ & 7 \\
\hline Overall antenna length in azimuth & $L_{a}$ & $11.2 \mathrm{~m}$ \\
\hline Transmit antenna size in azimuth & $d_{a z, t x}$ & $3.0 \mathrm{~m}$ \\
\hline $\begin{array}{l}\text { Reconstruction Network SNR } \\
\text { Scaling Factor }\end{array}$ & $\Phi_{b f}$ & $\leq 0.7 \mathrm{~dB}$ \\
\hline Receive aperture size in elevation & $d_{e l, r x}$ & $0.08 \mathrm{~m}$ \\
\hline Number of $\mathrm{Rx}$ apertures in elevation & $N_{e l}$ & 25 \\
\hline Overall antenna height in elevation & $H_{a}$ & $2 \mathrm{~m}$ \\
\hline Transmit antenna size in elevation & $d_{e l, t x}$ & $0.19 \mathrm{~m}-0.41 \mathrm{~m}$ \\
\hline Maximum transmit antenna gain & $G_{t x}$ & $38.7 \mathrm{~dB}-42.1 \mathrm{~dB}$ \\
\hline $\begin{array}{l}\text { Maximum gain of single receiver } \\
\text { channel }\end{array}$ & $G_{r x, j}$ & $46.2 \mathrm{~dB}$ \\
\hline Transmit peak power & $P_{t x}$ & $5 \mathrm{~kW}$ \\
\hline Duty cycle & $d c$ & $15 \%$ \\
\hline Boltzmann constant & $k$ & $1.38 \cdot 10^{-23} \mathrm{~J} / \mathrm{K}$ \\
\hline System temperature & $T$ & $300 \mathrm{~K}$ \\
\hline $\begin{array}{l}\text { Losses (atmospheric, system, } \\
\text { receiver noise, 2-way) }\end{array}$ & $L \cdot F$ & $5.7 \mathrm{~dB}$ \\
\hline Azimuth loss & $L_{a z}$ & $2.7 \mathrm{~dB}$ \\
\hline
\end{tabular}

line representing the swath's center indicates (cf. Fig. 7, bottom). When reaching the minimum PRF of $1250 \mathrm{~Hz}$, a "jump" to a PRF of $1470 \mathrm{~Hz}$ becomes necessary to guarantee continuous coverage (cf. Fig. 7, bottom, dotted line).

To achieve the required resolution in azimuth, simulations have shown that a processed bandwidth $B_{D}=7.6 \mathrm{kHz}$ is needed. In combination with the minimum PRF this determines a minimum number of receive apertures to ensure the necessary effective sampling ratio on receive. In the present case we obtain $N=7$. Further, the overall antenna length is to be chosen to set the optimum PRF within the required PRF range. It turns out that an overall length $L_{a}$ of $11.2 \mathrm{~m}=7 \cdot 1.6 \mathrm{~m}$ fulfils the requirements concerning the SNR scaling factor of the reconstruction network $\left(\Phi_{b f}\right)$ caused by the recovery of the nonuniform sampling. The respective value of $\Phi_{b f}$ resulting from the PRF for each swath is listed in Table III. An analysis of the ambiguous energy shows, that on transmit an aperture antenna of length $d_{a z, t x}=$ $3 \mathrm{~m}$ and without tapering results in a sufficiently high suppression (cf. Fig. 8, top). For a detailed analysis of the impact of different antenna lengths and tapering, refer to Section V. Table II summarizes the system parameters and dimensions.

To ensure a slant range resolution of $1 \mathrm{~m}$, a chirp bandwidth of $150 \mathrm{MHz}$ is necessary and hence the appropriate bandwidth $B_{R g}$ for a resolution of $1 \mathrm{~m}$ on ground is $150 \mathrm{MHz} / \sin \left(\theta_{i}\right)$, where $\theta_{i}$ denotes the incident angle. Consequently, the required 
bandwidth in range depends on the minimum $\theta_{i}$ of the respective swath. Further, to illuminate the whole swath for all incident angles, the effective height $d_{e l, t x}$ of the transmit antenna is varied to guarantee a coverage of $100 \mathrm{~km}$ on ground within the $3 \mathrm{~dB}$ beamwidth angle $\theta_{3 \mathrm{~dB}}$. The value of $\theta_{3 \mathrm{~dB}}$ in degrees is approximated by $51 \cdot \lambda / d_{e l, t x}$ according to [43]. The selection of the respective swath can be either achieved by an electrical steering of the transmit beam or a mechanical steering of the antenna by a roll-maneuver of the satellite. In the following, we assume a mechanical steering that ensures an optimum pointing of the antenna to the region to be imaged. Table III lists the respective values for the necessary chirp bandwidth, effective transmit antenna height, and corresponding maximum antenna gain $G_{t x, \max }$. On receive, the antenna in elevation consists of a large number of independent elements that allow for beamforming in elevation which ensures a high gain and a sufficient suppression of range ambiguities. For this, one may apply the scan-on-receive (SCORE) technique suggested in [14] and [15] that uses a real-time beamforming to scan the reflected pulse as it travels over the ground. Problems may occur with this technique by topography and it might be necessary to apply a more sophisticated approach to cope with the problems arising from height variations within the scene [44]. In order to guarantee the steering capability of the elevation beam to cover a swath of $100 \mathrm{~km}$, a scan angle up to $\pm 4.5^{\circ}$ with respect to antenna boresight is necessary. By scaling the solution for a swath width of $80 \mathrm{~km}$ in [35], the necessary element spacing in elevation to avoid grating lobes is chosen to $8 \mathrm{~cm}$. To obtain a sufficiently high gain on receive and achieve the required low NESZ, a receiving antenna of height of $2 \mathrm{~m}$ is used, which results in a number of 25 subantenna elements. Table II and Table III summarize the parameters in elevation dimension.

\section{System Performance: Azimuth Ambiguity Suppression, Resolution, NESZ}

In a first step, the suppression of azimuth ambiguities is simulated within the relevant PRF range and compared with its analytic prediction in (27) derived in Section IIIE. The results show good agreement with a deviation of less than $0.1 \mathrm{~dB}$
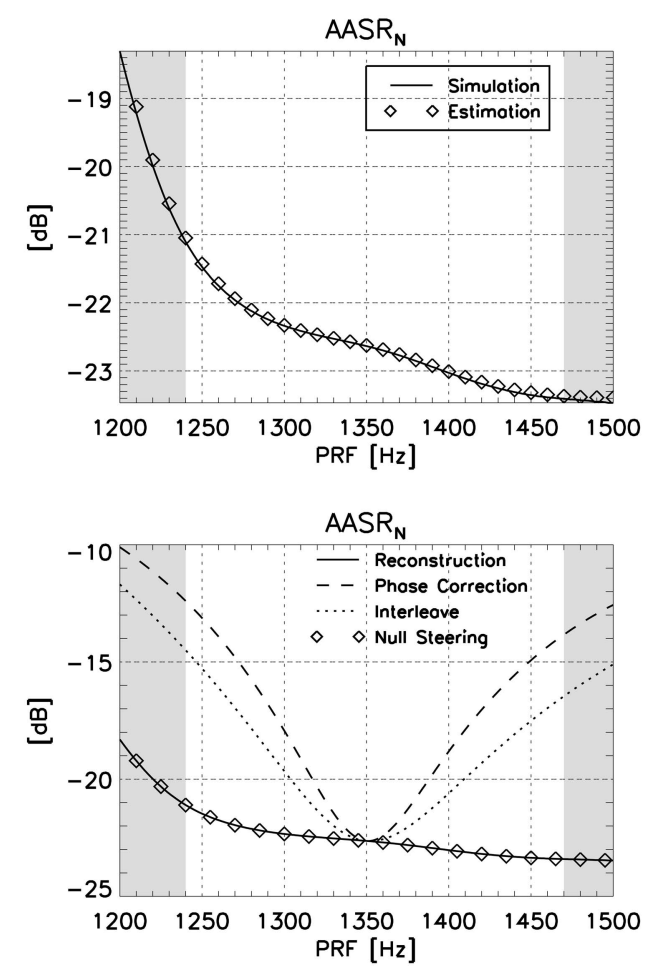

Fig. 8. Top: Simulated (solid) azimuth ambiguous energy suppression $\left(\mathrm{AASR}_{N}\right.$ ) versus $\mathrm{PRF}$ and its prediction (diamonds) for $B_{D}=7.6 \mathrm{kHz}$. Bottom: $\mathrm{AASR}_{N}$ achieved by reconstruction algorithm (solid) compared with phase correction (dashed) [24], simple interleaving of samples (dotted) [10], and pattern null-steering (diamonds) [33]. PRF region of interest limited by shaded areas.

(cf. Fig. 8, top). A closer analysis of (27) reveals that the amplification of the ambiguous energy outside the frequency interval $[-N / 2 \cdot \mathrm{PRF}, N / 2$. $\mathrm{PRF}]$ rises with increasing deviation from the optimum PRF, which is caused by the increasing mean squared value of the filter functions $P_{j}(f)$. Next, the multi-aperture reconstruction algorithm is compared with alternative algorithms for processing the multi-aperture azimuth signal. As a first reference case, the samples of the multiple azimuth channels are simply interleaved yielding the output signal without further processing as proposed in [10]. In a more complex approach, the method of [24] takes the properties of the SAR signal into account as it compares the multi-aperture signal's phase with the phase of a monostatic and uniformly sampled

TABLE III

Swaths and Respective Parameters

\begin{tabular}{ccccccr}
\hline \hline Swath & $\theta_{i}$ & $B_{R g}\left(\theta_{i, \min }\right)$ & $d_{e l, t x}$ & $G_{t x, \text { max }}$ & PRF & $\Phi_{b f}$ \\
\hline 1 & $20.0-29.0^{\circ}$ & $439 \mathrm{MHz}$ & $0.19 \mathrm{~m}$ & $38.7 \mathrm{~dB}$ & $1340 \mathrm{~Hz}$ & $-0.92 \mathrm{~dB}$ \\
2 & $27.5-35.6^{\circ}$ & $325 \mathrm{MHz}$ & $0.22 \mathrm{~m}$ & $39.4 \mathrm{~dB}$ & $1250 \mathrm{~Hz}$ & $0.06 \mathrm{~dB}$ \\
3 & $34.9-41.9^{\circ}$ & $262 \mathrm{MHz}$ & $0.26 \mathrm{~m}$ & $40.1 \mathrm{~dB}$ & $1350 \mathrm{~Hz}$ & $-0.96 \mathrm{~dB}$ \\
4 & $40.9-47.0^{\circ}$ & $229 \mathrm{MHz}$ & $0.30 \mathrm{~m}$ & $40.7 \mathrm{~dB}$ & $1260 \mathrm{~Hz}$ & $-0.12 \mathrm{~dB}$ \\
5 & $46.4-51.7^{\circ}$ & $207 \mathrm{MHz}$ & $0.36 \mathrm{~m}$ & $41.5 \mathrm{~dB}$ & $1330 \mathrm{~Hz}$ & $-0.86 \mathrm{~dB}$ \\
6 & $50.3-55.0^{\circ}$ & $195 \mathrm{MHz}$ & $0.41 \mathrm{~m}$ & $42.1 \mathrm{~dB}$ & $1260 \mathrm{~Hz}$ & $-0.12 \mathrm{~dB}$ \\
\hline
\end{tabular}




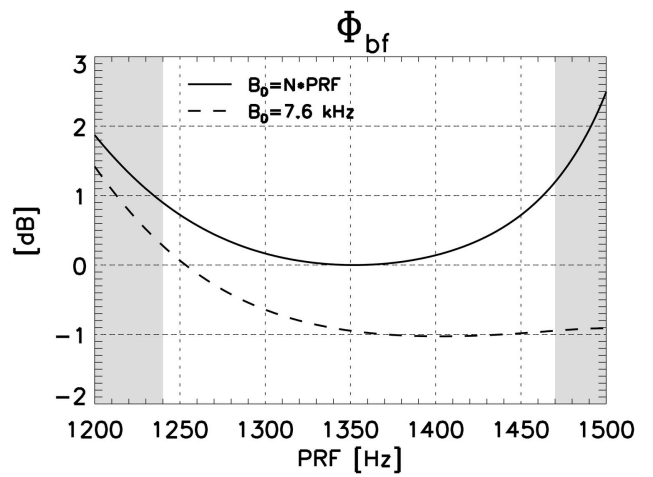

Fig. 9. Characteristic of SNR scaling factor $\Phi_{b f}$ versus PRF due to signal reconstruction with consideration of processed Doppler bandwidth of $7.6 \mathrm{kHz}$ (solid) and for whole bandwidth (dashed).

signal. This yields a Doppler frequency-dependent phase difference between the desired and the actual signal that is corrected by the processing. Finally, a space-time approach is evaluated that is based on a frequency-dependent adjustment of the weighting coefficients of the azimuth channels to steer nulls in the joint antenna pattern to the angles corresponding to the ambiguous Doppler frequencies. This null-steering corresponds to a spatial filtering of the data to suppress ambiguous frequencies in the azimuth signal $[27,33]$. Fig. 8 on the bottom shows that the best suppression is achieved by the reconstruction algorithm and the null-steering approach. As shown in Appendix C, under certain approximations this approach is already included in the reconstruction algorithm. For a more detailed comparison of different processing methods, refer to [36].

Regarding the azimuth resolution, all algorithms remain below $1 \mathrm{~m}$ for the optimum PRF of $1350 \mathrm{~Hz}$, but only the multi-channel processing approaches (phase correction, null-steering, reconstruction algorithm) provide a constant resolution over the whole PRF range, while the resolution degrades for increasing offset from the optimum PRF if no dedicated processing is applied and the samples are just interleaved.

Next, the noise equivalent sigma nought (NESZ) is determined. In a preliminary step, the SNR scaling factor $\Phi_{b f}$, which describes the variation of the SNR caused by the DBF network in dependency of the PRF, is evaluated (cf. Fig. 9). $\Phi_{b f}$ worsens with increasing deviation from the optimum PRF due to the rising mean square value of the $P_{j}(f)$ that tends to infinity when the PRF entails spatially coinciding samples and the whole system bandwidth in azimuth is considered. This effect is mitigated by the increasing oversampling caused by rising PRF values in combination with a constant $B_{D}$ entailing for the image a reduced part of the input noise power (cf. Fig. 9, solid line). The improvement for uniform sampling is directly given by $10 \cdot \log \left[B_{D} /(N \cdot \mathrm{PRF})\right]=$ $-0.95 \mathrm{~dB}$.
In addition, one has to account for the azimuth loss factor $L_{a z}$ that considers the decay of the joint $\mathrm{Tx} / \mathrm{Rx}$ azimuth pattern which attenuates the recorded signal while the added noise power remains spectrally white [45]. Hence, the SNR becomes dependent on the Doppler frequency. Assuming a normalised rectangular filter of bandwidth $B_{D}$ for focusing in azimuth, $L_{a z}$ is expressed by (39), where $A_{0}(f)$ represents the normalised weighting of the Doppler spectrum by the joint Tx/Rx azimuth antenna pattern. A transmit antenna of $3 \mathrm{~m}$ and a receiving antenna of $1.6 \mathrm{~m}$ yield a loss of $L_{a z}=2.7 \mathrm{~dB}$

$$
L_{a z}=\frac{B_{D}}{\int_{-B_{D} / 2}^{B_{D} / 2}\left|A_{0}(f)\right|^{2} d f} .
$$

Finally, the well-known expression for the NESZ [1] is extended by the SNR scaling factor of the reconstruction filter network $\Phi_{b f, B_{D}}$ (cf. Section IIIF) which comprises a possible gain by oversampling, but does not account for the changed input $\mathrm{SNR}_{e l}$ with the PRF. As $\Phi_{b f, B_{D}}$ is normalized to $\mathrm{PRF}_{\text {uni }}$, an additional compensation factor $\Phi_{b f, \mathrm{NESZ}}=\mathrm{PRF} / \mathrm{PRF}_{\text {uni }}$ is required. Effectively, this considers the noise power at PRF with respect to $\mathrm{PRF}_{\text {uni }}$ according to the changed number of samples. Note that all other losses (system, atmospheric, etc.) and the receiver noise figure are summarized in the loss factor $L \cdot F$. The description and values of all parameters can be found in Table II

$$
\mathrm{NESZ}=\frac{\begin{array}{c}
256 \cdot \pi^{3} \cdot R_{0}^{3}\left(\Theta_{i}\right) \cdot v_{s} \cdot \sin \left(\Theta_{i}\right) \\
\cdot k \cdot T \cdot B_{r g}\left(\Theta_{i}\right) \cdot \Phi_{b f, B_{D}} \Phi_{b f, \mathrm{NESZ}} \cdot L \cdot F \cdot L_{a z}
\end{array}}{P_{t x} \cdot G_{t x}\left(\Theta_{i}\right) \cdot N \cdot G_{r x, j} \cdot \lambda^{3} \cdot c_{0} \cdot d c} .
$$

Under consideration of the above parameters and the respective transmit pattern we obtain the following characteristic of the NESZ versus ground range (cf. Fig. 10, top). In the case where an arbitrary swath of $100 \mathrm{~km}$ can be imaged, the variation of the NESZ for a swath centred at a certain range is given by the best and worst value within the swath (cf. Fig. 7, bottom). Note that this takes into account the adaptation of the chirp bandwidth and the effective transmit antenna height with varying ground range. The steps in the curve result from the steps in the PRF and the corresponding SNR scaling factor of the reconstruction network. In both cases the NESZ is below the required $-19 \mathrm{~dB}$.

\section{OPTIMIZATION POTENTIALS}

In a further step, strategies for optimizing the SAR system performance are developed. The first section identifies the main error sources, analyzes their impact on the respective performance parameters and derives general strategies to minimize the errors. Then, in the following sections, specific techniques according to those strategies are developed and the 

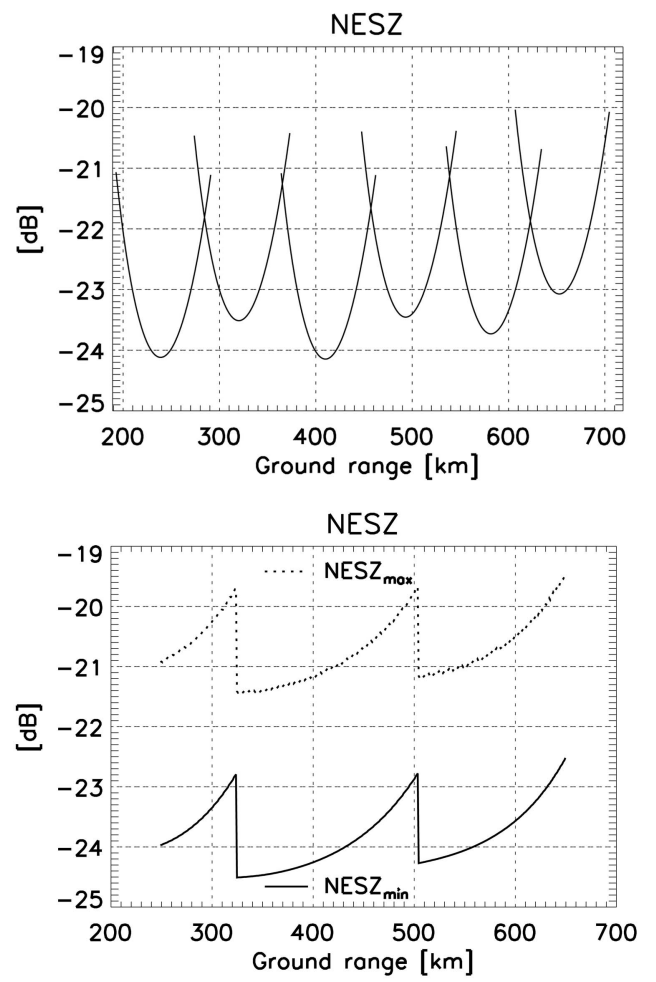

Fig. 10. Top: NESZ versus ground range for coverage by 6 distinct swaths. Bottom: Best (solid) and worst (dotted) NESZ for arbitrarily located swaths. Ground range gives pointing location of transmit antenna beam.

results of the optimized system are presented. As main performance parameters to be optimized, we focus on the signal-to-noise ratio, suppression of ambiguous energy, and geometric resolution. The optimization aims at improving the SNR and the ambiguity suppression while keeping the resolution at a constant level.

\section{A. Error Sources and Optimization Approaches}

1) Error Sources: In Section IIIE it was presented that the ambiguous energy in the signal after reconstruction is determined by two aspects. First (as in any conventional SAR system) the joint antenna characteristic of transmit and single receive aperture contains information from azimuth angles that correspond to Doppler frequencies outside the band $I_{s}=[-\mathrm{PRF} \cdot N / 2, \mathrm{PRF} \cdot N / 2]$. These contributions are subsampled and give rise to azimuth ambiguities in the image. In addition, the ambiguous energy is weighted and possibly amplified by the power spectral densities of the reconstruction filter functions $P_{j m}(f)$. In general, this amplification is more severe, the stronger the nonuniform sampling of the signal is. Concerning the noise power, Section IIIF showed that the processing by the reconstruction filter functions may cause a degradation of the SNR, as the filters $P_{j m}(f)$ possibly amplify the noise power while preserving the signal power. Analogically to the ambiguities, this amplification rises with increasing nonuniform sampling of the signal due to the inverse character of the filter system. In this context, an investigation of the spectral properties of $\Phi_{b f}$ shows that the degradation of the SNR for strong nonuniform sampling with PRF values above the optimum PRF is dominated by the spectral sub-bands $m$ of order 1 and $N$, i.e., the lowest and highest sub-band within the system bandwidth $I_{s}$. Consequently, the singularity of the noise and ambiguity scaling for spatially coinciding samples (cf. Section IVC) is caused by these sub-bands, while the contribution of the other sub-bands is not critical. This seems logical from an information theoretical point of view as the remaining samples when skipping the coinciding channels still yield an effective sampling ratio that fulfils the Nyquist criterion with respect to the bandwidth of the inner sub-bands.

2) Optimization Potential: The above analysis of error sources yields two main areas of possible optimization. First, the minimization of ambiguous energy in the received signal allows for reducing the ambiguities in the image. This means that antenna patterns on transmit and receive are adapted to optimally confine the desired Doppler band, which is the subject of a detailed analysis following in Section B that introduces the idea of tapering on transmit, while Section $\mathrm{C}$ includes the adaptation of the receive characteristic. Besides, the azimuth processing shows potential for improving the performance by minimizing the error amplification caused by the reconstruction filters. In a first simple approach, the ratio between PRF and $B_{D}$ can be set in a way to benefit from a large oversampling and allow for eliminating frequency bands that primarily cause the degradation of the performance. Basically this corresponds to an appropriate lowpass filtering of the signal to achieve acceptably moderate levels of SNR degradation even near to singular PRFs. According to the first paragraph it is necessary to filter the $n$ outermost bands at the lower and upper border of the Doppler spectrum to suppress completely the strong rise in noise scaling at the $n$th singular PRF. Hence, the sampling ratio $N$. PRF has to exceed the processed bandwidth $B_{D}$ by a factor of $N /(N-2 \cdot n)$ to keep the SNR degradation low:

$$
B_{D} \leq(N-2 \cdot n) \cdot \mathrm{PRF} .
$$

Although a very effective way of reducing the SNR degradation, this requires a relatively high PRF to guarantee the necessary oversampling with respect to the processed bandwidth $B_{D}$. This might cause problems with the timing. Simply decreasing $B_{D}$ for a given PRF represents the easier way to ensure (41), but is at the cost of a deteriorated geometric resolution. Nevertheless, a strongly improved SNR might be obtained by a moderately degraded resolution. So, finally this results in a trade-off between azimuth resolution and SNR. 


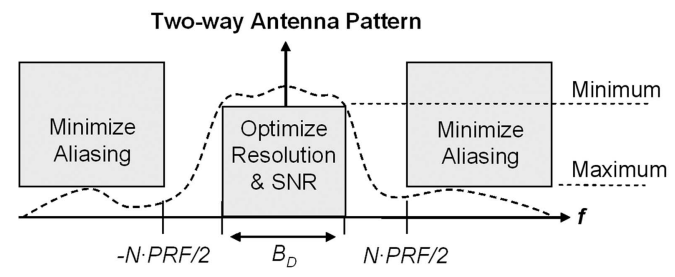

Fig. 11. Basic principle of pattern tapering on transmit. Suppression of aliased energy in combination with optimization of signal energy.

Furthermore one can aim at improving the conditions for the processing by matching the phase centres and the PRF to obtain a data array that is sampled as uniform as possible. This can be either achieved by an adaptive management of the PRF to obtain an improved spatial sampling, but this approach shows only great potential in sparse array systems and is hence only treated shortly in Appendix D. In single platform systems, it is more promising to adapt the phase centers to the PRF by adding a reconfigurable preprocessing network to the conventional beamforming obtaining a cascaded network as introduced and elaborated in Section C.

In a next stage, the processing itself might be modified, mitigating the negative effects of the inverse filter by introducing a trade-off between ambiguity suppression and SNR optimization in the processing strategy. This comprises for example an adapted beamforming approach that merges the classical ambiguity suppression strategy with a beam steering approach that optimizes the signal power (cf. Section D). As one will see, the transition from the techniques in Section C to Section D is smooth, as both approaches are based on cascaded processing networks.

\section{B. Pattern Tapering on Transmit}

As derived in Section IIID, all spectral energy outside the band $[-N \cdot \mathrm{PRF} / 2, N \cdot \mathrm{PRF} / 2]$ causes aliasing in the reconstructed signal and finally gives rise to ambiguities. This can be avoided by confining the Doppler bandwidth of the signal to $N$. PRF by an appropriate antenna pattern. In a very simple approach, one could just enlarge the dimension of the transmit antenna resulting in a narrower pattern. But as this is achieved only at the expense of resolution, a bigger antenna in combination with an adapted tapering is to be used to provide improved ambiguity suppression without degrading the resolution.

Furthermore, the better the pattern is limited to the relevant Doppler frequencies, the better the emitted power is used as less power is lost by illuminating unwanted areas and consequently the NESZ of the system is improved. The basic idea is visualized in Fig. 11.

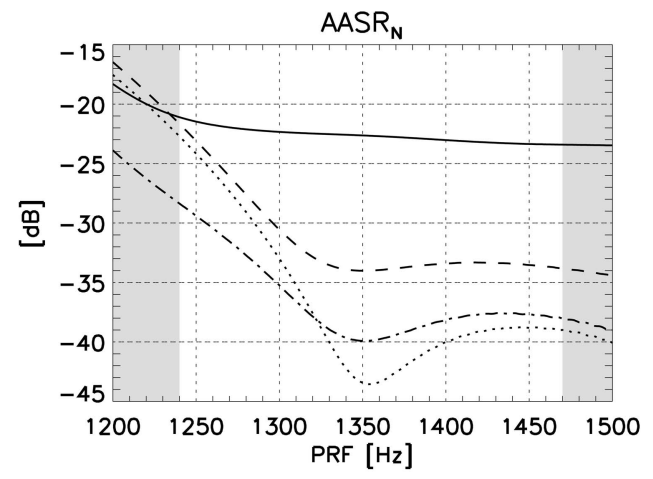

Fig. 12. $\mathrm{AASR}_{N}$ versus PRF for $B_{D}=7.6 \mathrm{kHz}$ and different transmit antenna sizes and patterns. $\sin (f) / f$ characteristic for an aperture of $3 \mathrm{~m}$ (solid), $4 \mathrm{~m}$ antenna with $\cos (x)$ excitation (dashed), $4.6 \mathrm{~m}$ antenna with $(\sin (f) / f)^{2}$ characteristic (dotted) and approximately rectangular pattern (dotted dashed) for $11.2 \mathrm{~m}$ antenna.

To demonstrate the potential of pattern tapering on transmit, we consider the system of Section IV and investigate different combinations of transmit antenna dimensions and excitations that can be either realized by a separate transmit antenna or by using an active array that offers the flexibility to use parts of the receiving antenna for transmit. Fig. 12 shows the results for the azimuth ambiguity suppression $\mathrm{AASR}_{N}$ in comparison to the $3 \mathrm{~m}$ transmit antenna with a uniform taper and hence a $\sin (f) / f$ pattern (solid) as used in Section IV. The suppression is already clearly improved for higher PRF values by applying a $4 \mathrm{~m}$ antenna with a $\cos (x)$ excitation (dashed) and becomes even better with a $4.6 \mathrm{~m}$ antenna with a simple triangular tapering which entails a $(\sin (f) / f)^{2}$ characteristic (dotted). However, to provide an improvement in suppression also for lower PRF values, a larger transmit antenna is necessary. An unconventional realization is given by the quasi-optimum - as it approximates a rectangular pattern $-\sin (x) / x$ excitation in combination with a transmit antenna of $11.2 \mathrm{~m}$ (dotted dashed) what corresponds to the antenna length on receive. It is realized by 35 elements of $0.32 \mathrm{~m}$ length each. Especially the $\sin (x) / x$-excitation demonstrates the potential of tapering to efficiently cancel the spurious spectral components while preserving the resolution. Note that all scenarios show a resolution below $1 \mathrm{~m}\left(B_{D}=7.6 \mathrm{kHz}\right)$. A full exploitation of the benefits of tapering requires a fine adjustment of antenna dimensions, PRF and $B_{D}$, taking into account the trade-off between resolution and ambiguity suppression.

In the frame of advanced concepts of transmit antenna architectures in azimuth, a next step comprises an antenna that allows for adaptively varying the transmit phase center from pulse to pulse by using only certain parts of the antenna. This would enable a sliding phase center on transmit from pulse to pulse that has the potential to compensate for 


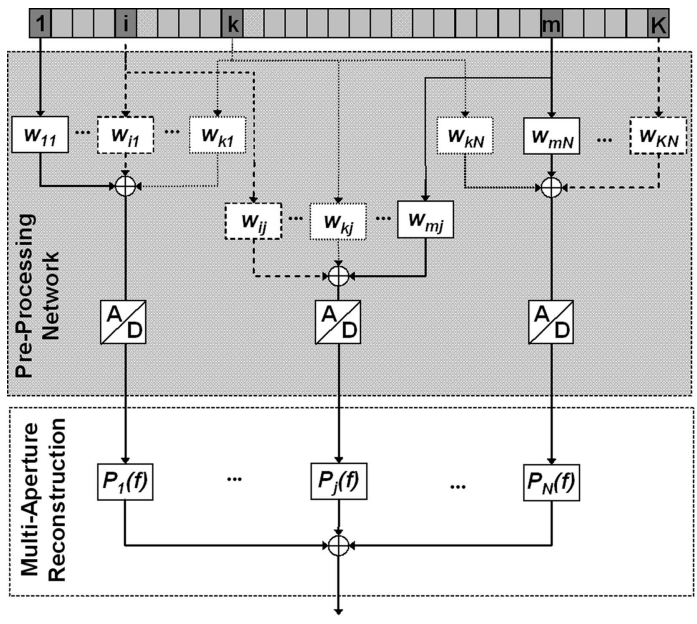

Fig. 13. "Prebeamshaping on receive" network as analogue representation of preprocessing network. Amplified RF signals of various apertures are adaptively weighted and combined before A/D conversion. This yields $N$ optimized output channels that enter the following DBF network.

a nonoptimum PRF. By choosing the direction of the sliding phase center along or against the flight direction, it is possible to adjust the spatial sampling resulting from too high or too low PRF values, respectively. The PRF range that can be compensated is determined by the maximum displacement of the phase center, while the fine-tuning ability of the position, i.e., the "step size" of the phase center from pulse to pulse, depends on the distance between single transmit elements. Note that a shift of the phase center is necessary every time it has reached its outermost position. In the case of a too high PRF this requires combining or even skipping some samples that might overlap while in the case of a too low PRF this results in "missing" samples within the synthetic aperture, as a gap occurs when the phase center is switched.

\section{Cascaded Beamforming Networks I-Analogue Prebeamshaping on Receive}

In the context of system optimization more sophisticated beamforming on receive approaches are regarded as powerful tools to adaptively improve the system performance. In a cascaded beamforming network the existing system is extended by a second network that is used for analogue or digital preprocessing of the multi-aperture SAR signals before the reconstruction filter network introduced in Section III is passed. In this section, we stick to an analogue preprocessing technique of the multi-aperture RF signals, a so-called prebeamshaping on receive network, but the results are also valid for a digital preprocessing step introduced in Section D. The system is based on an antenna consisting of a number of $K$ independent elements. Then a network follows, allowing for an individual and reconfigurable weighting and combination of the elements' signals
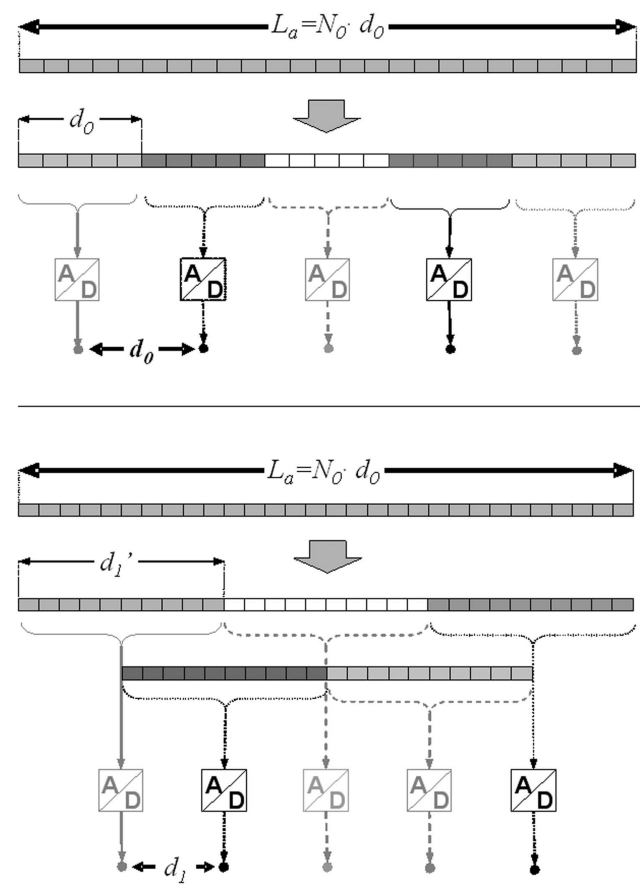

Fig. 14. Conventional multi-aperture system with subaperture size $d_{0}$ equal to the phase center distance (top) and system with prebeamshaping on receive network (bottom). The multiple use of certain elements yields mutually overlapping subapertures of increased length $d_{1}^{\prime}$, but decreased phase center spacing $d_{1}$.

resulting in $N$ "virtual" output channels $j$. The weights may vary from subaperture to subaperture and from channel to channel. This allows for "using" the signal of a certain subaperture in more than one of the virtual channels resulting in a spatial overlap of these channels. The contribution of the signal of element $i$ to the virtual channel $j$ is described by the complex coefficient $w_{i j}$ (cf. Fig. 13). In the case of analogue preprocessing, each signal is amplified separately by an LNA before weighting, combining, and digitizing the RF signals.

The basic idea behind the network is to modify the received signal in way to match it to the reconstruction network, primarily by flexibly setting the number and phase center position of the virtual channels that are formed. This allows for adjusting the spatial sample positions to the actual system PRF to minimize the nonuniform sampling. In addition, such networks enable to implement pattern tapering on receive thus offering a powerful tool to e.g. suppress ambiguities. The reconfigurable structure allows for flexibly allocating the network resources to emphasize a specific system parameter that finally results in an adjustable trade-off between SNR, $\mathrm{AASR}_{N}$, and resolution.

In the following the basic relations regarding the formation of virtual channels are presented to demonstrate the idea and potential of the prebeamshaping concept. Consider a multi-aperture antenna of overall length $L_{a}=N_{0} \cdot d_{0}$, where $d_{0}$ 
represents the length of the aperture that forms the single channel. The spacing between the phase centers is equal to the length of the subapertures and hence given by $d_{0}$, which determines the optimum PRF (cf. Fig. 14, top). In Fig. 14, on the bottom, a simple realization of a prebeamshaping network is shown, where some of the receiving elements are part of multiple virtual channels yielding a number of $N$ mutually overlapping subapertures of length $d_{1}^{\prime}>d_{0}$. Due to the overlap of adjacent apertures, the resulting phase center spacing $d_{1}$ decreases with respect to the reference case shown on the top, where no prebeamshaping network is applied, i.e., $d_{1}<d_{0}$. Consequently while keeping the overall antenna length constant, the resulting spatial sampling of the system is modified as the corresponding optimum PRF is now defined by $N$ and $d_{1}$. This means that one obtains a system that allows for flexibly setting the phase centers appropriate to the operating PRF and improving the SNR. In this context, the modified SNR scaling factor of a cascaded beamforming networks has to be taken into account as we see in the following. In addition, a larger subaperture length is available enabling pattern tapering of the receiving apertures by adjusting the network's weighting coefficients. The enlarged subaperture length $d_{1}^{\prime}$ and the corresponding phase center distance $d_{1}$ are connected by (42) and define the overlap $d_{o v}$ of adjacent apertures as given in (43), resulting in a new optimum $\mathrm{PRF}_{\text {uni,c }}$ given by (44)

$$
\begin{aligned}
d_{1} & =\frac{L_{a}-d_{1}^{\prime}}{N-1}=\frac{N_{0} \cdot d_{0}-d_{1}^{\prime}}{N-1} \\
d_{o v} & =d_{1}^{\prime}-d_{1} \\
\mathrm{PRF}_{\mathrm{uni}, c} & =\frac{2 \cdot v_{s}}{N \cdot d_{1}}=\frac{N_{0} \cdot(N-1)}{N \cdot\left(N_{0}-d_{1}^{\prime} / d_{0}\right)} \cdot \mathrm{PRF}_{\mathrm{uni}} \cdot
\end{aligned}
$$

Note that the preprocessing network has an impact on signal, ambiguities and noise power and thus the expressions describing these parameters that were derived in Sections IIID-IIIF have to be adapted to the extended beamforming scenario. The following paragraph briefly summarizes the differences to the conventional beamforming network and the resulting relations that are derived in detail in Appendix A. The relevant signal for the output signal power is determined by the input signals of the reconstruction network (cf. Section IIID). These signals are given by the $N$ output signals of the prebeamshaping network, which are defined by the weighted sum of signals received by the single elements. The respective weighting is given by the coefficients $w_{i j}(f)$, that can be Doppler frequency dependent in the case of a digital preprocessing network (cf. Section VD). Further, similar to Section IIIA, the received signal at a single element can be modeled as an equivalent monostatic signal $U(f)$ filtered with an appropriate function $M_{i}(f)$ that takes into account the offset between transmitter and receiving element. Further, in an analogue implementation the need for power dividers will result in amplitude imbalances in the signals that have to be compensated for by adapting the weighting functions $w_{i j}$ according to (52) (cf. Appendix A). This allows for setting up the following relation between input signal power $p_{s, e l}$ and output signal power before and after focusing, given by $p_{s, c}$ in (45) and $p_{s, c, B D}$ in (46), respectively

$$
\begin{aligned}
p_{s, c} & =p_{s, e l} \cdot N^{2} \cdot G \cdot \mathbf{E}\left[\left|\sum_{i=1}^{K}\left(M_{i}(f) \cdot w_{i j}(f)\right)\right|^{2}\right] \\
p_{s, c, B_{D}} & =N^{2} \cdot G \cdot \mathbf{E}\left[A(f)^{2} \cdot\left|\sum_{i=1}^{K} M_{i}(f) \cdot w_{i j}(f) \cdot \operatorname{rect}\left(f / B_{D}\right)\right|^{2}\right] .
\end{aligned}
$$

In a similar way, the power of the ambiguities is to be adapted to the prebeamshaping scenario. This means that all expressions derived in Section IIIE can be adapted to the extended processing network by adding the power gain $G$ of the LNA and replacing $U(f)$ with the resulting equivalent monostatic output signal of the prebeamshaping network $U_{c}(f)$, which is derived in Appendix A and represents the spectral appearance of the signals before the reconstruction network, but takes into account the prebeamshaping on receive.

Finally, let us consider the influence of the prebeamshaping network on the output noise power $p_{n, c}$ under the assumption of a lossless network of weighting elements $w_{i j}(f)$. As mentioned before, the receiver noise is assumed to be mutually uncorrelated additive white Gaussian noise. This means that the input noise power $p_{n, e l}$ is amplified by the prebeamshaping network and the following reconstruction, yielding the output noise power in the data $p_{s, c}$ and in the image $p_{s, c, B D}$, according to (47) and (48), respectively. Note that in contrast to the mere DBF network discussed before, the mutual correlations introduced by the prebeamshaping do not allow any more for simplifying the squared sum to a sum of squares

$$
\begin{aligned}
p_{n, c} & =p_{n, e l} \cdot G \cdot F \cdot \sum_{i=1}^{K} \mathbf{E}\left[\left|\sum_{j=1}^{N} P_{j}(f) \cdot w_{i j}(f)\right|^{2}\right] \\
p_{n, c, B_{D}} & =p_{n, e l} \cdot G \cdot F \cdot \sum_{i=1}^{K} \mathbf{E}\left[\left|\sum_{j=1}^{N} P_{j}(f) \cdot w_{i j}(f) \cdot \operatorname{rect}\left(f / B_{D}\right)\right|^{2}\right] .
\end{aligned}
$$

Combining the expressions for signal power (cf. (45)) and noise power (cf. (47)) and normalizing by the optimum value $F / K$ yields the SNR scaling factor 


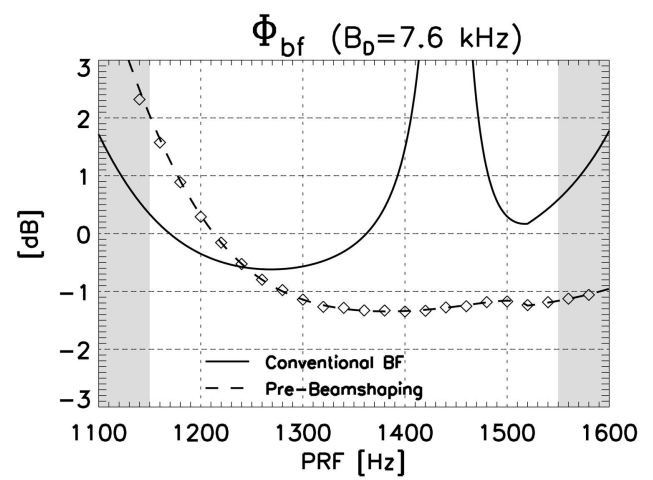

Fig. 15. Predicted SNR scaling factor $\Phi_{b f}$ of the the conventional DBF network (solid) compared with the case where prebeamshaping network is added (dashed). Simulated values overlaid in diamonds $\left(B_{D}=7.6 \mathrm{kHz}\right)$.

$\Phi_{b f, c}$ of the cascaded network with respect to the data (cf. (49)). As before, the SNR scaling factor with respect to the image cannot be given explicitly, but can be determined by calculating the signal power $p_{s, c, B D}$ and the noise power $p_{n, c, B D}$ according to (46) and (48), respectively

$$
\begin{aligned}
\Phi_{b f, c} & =\frac{p_{n, c} / p_{n, e l}}{p_{s, c} / p_{s, e l}} \cdot \frac{K}{F} \\
& =\frac{K \cdot \sum_{i=1}^{K} \mathbf{E}\left[\left|\sum_{j=1}^{N} P_{j}(f) \cdot w_{i j}(f) \cdot \operatorname{rect}\left(f / I_{S}\right)\right|^{2}\right]}{N^{2} \cdot \mathbf{E}\left[\left|\sum_{i=1}^{K} M_{i}(f) \cdot w_{i j}(f) \cdot \operatorname{rect}\left(f / I_{S}\right)\right|^{2}\right]} .
\end{aligned}
$$

As explained in detail in Appendix A, the quantization noise power in an analogue realization $p_{n, q, a}$ is described by (50), while (51) in Section D will give the noise resulting from a digitization before the preprocessing network. The comparison of (50) with (51) implies that digitizing as late as possible reduces the scaling of the quantization error

$$
p_{n, q, a}=\mathbf{E}\left[\left|\sum_{j=1}^{N} P_{j}(f) \cdot n_{q j}(f)\right|^{2}\right] \text {. }
$$

To demonstrate the potential of the prebeamshaping on receive technique we assume a modified system where each subaperture consists of multiple independent elements. With respect to the system presented in Section IV, the new system is designed to be applicable in a larger PRF range from 1150 to $1550 \mathrm{~Hz}$. Hence, the subaperture length is increased to $1.75 \mathrm{~m}$ resulting in an overall length of $12.25 \mathrm{~m}$ and a decreased optimum PRF of $1234 \mathrm{~Hz}$. Further, a slightly increased aperture antenna on transmit of $3.15 \mathrm{~m}$ is required to ensure a sufficient ambiguity suppression as we see later. All other system parameters remain unaltered.

An analysis of the SNR scaling factor $\Phi_{b f}$ of

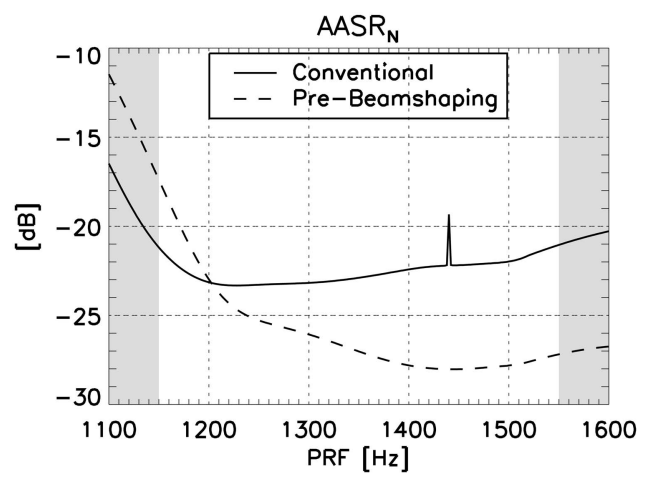

Fig. 16. $\mathrm{AASR}_{N}$ versus PRF with prebeamshaping network (dashed) compared with conventional DBF (solid) $\left(B_{D}=7.6 \mathrm{kHz}\right)$. Peak in conventional approach occurs at "singular" $\mathrm{PRF}=1440 \mathrm{~Hz}$ (cf. Section IIC).

the reconstruction network shows sufficiently low values from $1150 \mathrm{~Hz}$ up to approximately $1350 \mathrm{~Hz}$, but yields unacceptably high values for the PRF range above (cf. Fig. 15, solid line). To provide a low SNR scaling over the complete PRF range, a reconfiguration of the prebeamshaping network becomes necessary for higher PRF values. In the present case, mutually overlapping subapertures of $2.625 \mathrm{~m}$ length are formed, resulting in a decreased phase center spacing that corresponds to a new optimum PRF of $1346 \mathrm{~Hz}$. In order to keep the beamwidth of the receiving pattern constant and to suppress its sidelobes, a cosine taper is applied to each of the subapertures. The respective $\Phi_{b f, c}$ is given in Fig. 15 (dashed line). It is normalised to the optimum $\Phi_{b f}$ of the conventional network and takes into account the impact of the subaperture dimension and taper on noise power, signal peak power, and azimuth loss $L_{a z}$ that has increased to $3.05 \mathrm{~dB}$ due to the preprocessing network compared with $L_{a z}=2.9 \mathrm{~dB}$ in the conventional case. Fig. 15 shows that an improved SNR scaling factor is obtained for PRF values above $1240 \mathrm{~Hz}$.

In terms of the NESZ, we focus on the differences of the modified system to the system of Section IV. First, one can state a degradation of $0.2 \mathrm{~dB}$ due to the increased $L_{a z}$. It results from an increased transmit antenna and an increased receive aperture giving rise to an overall $L_{a z}$ of $2.9 \mathrm{~dB}$ compared with $2.7 \mathrm{~dB}$ of the original system. In contrast, the new antenna dimensions result in an increased gain of $0.2 \mathrm{~dB}$ on transmit and $0.4 \mathrm{~dB}$ on receive and the SNR scaling factor of the reconstruction network (cf. Fig. 15) tends to be better than in the original system (cf. Fig. 9), which might be compensated by the decreasing $\mathrm{SNR}_{e l}$ with increasing PRF according to (40). Altogether, the NESZ of the original system (cf. Fig. 10) gives a good estimation for the new NESZ.

Concerning the resolution, the increased subaperture length of $1.75 \mathrm{~m}$ with respect to the length of $1.6 \mathrm{~m}$ in the original system entails a 
minor degradation to $1.03 \mathrm{~m}$, both for conventional and prebeamshaping system operation. Finally, the ambiguous energy suppression of the modified system is investigated. As mentioned above, the transmit antenna was slightly increased to $3.15 \mathrm{~m}$ to guarantee a minimum suppression of $-21 \mathrm{~dB}$ (cf. Fig. 16). When the preprocessing network is applied, the suppression becomes better for PRF values above $\sim 1200 \mathrm{~Hz}$ and is clearly improved for higher PRF values due to reduced sidelobes of the receive pattern and minimized amplification of the azimuth ambiguous energy caused by the adapted phase centers (cf. Fig. 16, dashed line). In combination with the results for the SNR scaling factor $\Phi_{b f}$, it is hence favourable to use the conventional configuration below a PRF of $1200 \mathrm{~Hz}$ and apply the prebeamshaping up from $1240 \mathrm{~Hz}$. In between the focus can be either turned to the NESZ by choosing the conventional approach or the preprocessing is applied to concentrate on the optimization of the $\operatorname{AASR}_{N}$.

\section{Cascaded Beamforming Networks II-Adapted Digital Beamforming}

Similar to the prebeamshaping network presented before, this approach is based on subsequent processing networks, but in contrast to the analogue prebeamshaping, all signals are sampled, digitized, and stored before processing. Hence, the amount of data to be handled limits in this case the maximum number of individual elements but a complete a posteriori processing becomes possible enabling wide flexibility and reconfigurability in terms of order and structure of the networks. As the basic principle is again a cascaded structure of networks, the expressions for the SNR scaling factor $\Phi_{b f}$ in (45)-(49) are valid.

Recall the "classical" reconstruction algorithm presented in Section III that aims at recovering the unambiguous Doppler spectrum by suppressing the ambiguous frequency components. In terms of the group antenna characteristic that results from the weighting coefficients of the beamforming algorithm, this corresponds to placing nulls at angles where ambiguous Doppler frequencies are situated. Further, one observes that only for uniform sampling, the maximum in the group characteristic is steered at the Doppler frequency that is to be recovered. For increasing nonuniformity, the maximum drifts away from that angle resulting in a decreased gain. Hence, the degradation of the SNR is caused by a loss of the signal energy that is compensated for by a later scaling of the signal that lifts up the noise floor. A basic idea of the "adapted beamforming" by cascaded networks is now to introduce a trade-off between ambiguity suppression and optimized signal energy to improve the SNR. This means that a number of degrees of freedom is used for placing nulls in the group pattern while other weights are chosen to optimize the signal power. Basically this approach results in the cascaded structure of two subsequent networks where one effectuates the steering of the mainlobe while the other cancels the azimuth ambiguities. The order of the networks and the combinations of the subapertures are in principle arbitrary as long as the structure allows for the multiplication of the respective patterns of the two stages. This means that the weighting functions are the same for each of the formed channels. Such networks show flexibility with respect to the receiving pattern and the effective sampling ratio given by the number of different reconstructed channels. Further, the spatial sample positions can be adapted, but only with certain flexibility as this is directly linked to the number of reconstructed channels. Regarding SNR, $\mathrm{AASR}_{N}$, and resolution this results in a trade-off between the effective sampling ratio, the receiving pattern, and an eventual amplification by the reconstruction algorithm depending on the spatial sampling.

Concerning the scaling of the quantization noise, the position of the A/D converter with respect to the weighting networks has to be considered, as only processing steps after the digitization affect the quantization error. This means that in contrast to (50) the preprocessing is taken into account for determining the power of the quantization noise $p_{n, q, d}$

$$
p_{n, q, d}=\sum_{i=1}^{K} \mathbf{E}\left[\left|\sum_{j=1}^{N} P_{j}(f) \cdot w_{i j}(f) \cdot n_{q i}(f)\right|^{2}\right] \text {. }
$$

One possible realization of a cascaded network structure is given by Fig. 17, where the multi-aperture reconstruction is followed by a beam-steering with weights $w_{i}(f)$. Consider an antenna of $N_{0}$ receiving elements, where $N$ channels are processed by the multi-aperture reconstruction algorithm, respectively, to form $\mathrm{N}_{2}$ output channels that may mutually overlap or not. The resulting $N_{2}$ channels are then weighted and combined where the weighting functions $w_{i}(f)$ are chosen to steer the maximum of the joint antenna characteristic for every $f \in\left[-B_{D} / 2, B_{D} / 2\right]$ in the direction corresponding to that frequency. As the multi-aperture reconstruction processing does not affect the signal envelope, the beamwidth of the output signal is defined by the single element length, while the gain is determined by the number of channels that are combined in the second stage. This means that the signal amplitude gain rises by a factor of $N_{2}$ at the cost of an effective sampling ratio that is decreased by a factor of $N_{0} / N$ and an increased noise floor. The latter is caused by mutual correlations between the noise components introduced by partly overlapping output channels. Equations (45)-(49) 


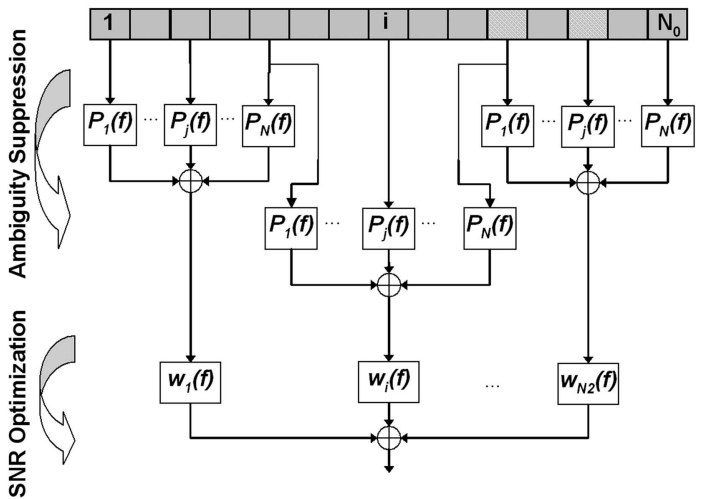

Fig. 17. Cascaded networks for ambiguity suppression followed by beam-steering with mutual overlap of adjacent filter groups of first stage.

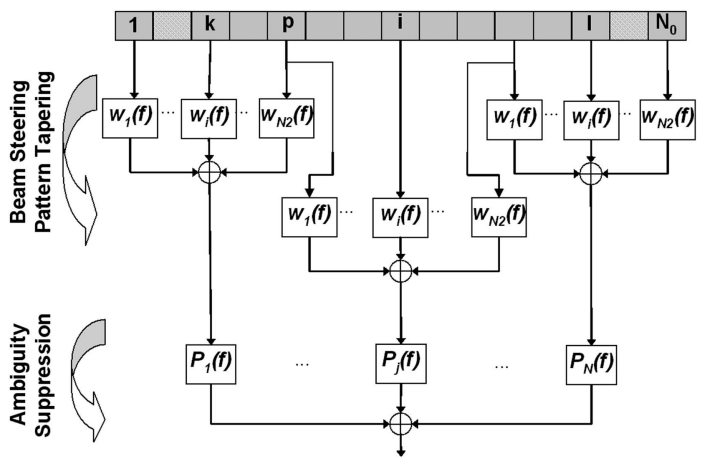

Fig. 18. Cascaded networks: Beam-steering and pattern tapering followed by reconstruction of $N$ channels with mutual overlap of adjacent beam-steering groups. This representation is similar to prebeamshaping network but with less elements and A/D conversion carried out before processing.

describe the resulting SNR scaling factor of the network.

The commutativity of the system allows for changing the different stages as long as the resulting processing of each element's branch remains unaltered. Consequently, the system of Fig. 17 can be equivalently represented by inversely ordered networks. Fig. 18 shows an example of such a structure where the steering of the beam in combination with a possible tapering is carried out before multi-aperture reconstruction.

One recognizes that the way of combining the two stages offers a wide range of flexibility, for example a closer spacing of the resulting phase centers can be achieved by subapertures that are no longer formed by adjacent but distributed elements. The minimum possible phase center spacing is given by the distance between adjacent elements. It can be achieved if subapertures made up of $N_{2}$ elements overlap by $N_{2}-1$ elements with their neighbored subapertures or if distributed elements form the subapertures. The maximum spacing is obtained in the conventional case where adjacent subapertures do not overlap. In combination with the number of channels that are formed, this offers a wide range of adaptation

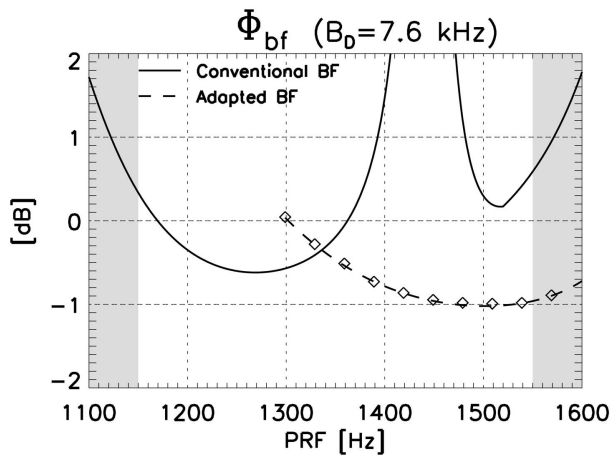

Fig. 19. Prediction (dashed) and simulation (diamond symbols) of SNR scaling factors $\Phi_{b f}$ for adapted beamforming compared with prediction of conventional DBF (solid).

regarding the PRF. Note that all processing is applied a posteriori and consequently the system setting can be reconfigured arbitrarily to focus on the respective performance parameter of interest, be it ambiguity suppression, resolution, or NESZ.

In the following, the potential of the adapted beamforming approach is demonstrated with a system example. Consider again the system of Section C with 7 subapertures of length $1.75 \mathrm{~m}$, each, and a PRF range of operation from $1150 \mathrm{~Hz}$ to $1550 \mathrm{~Hz}$. As derived in Section C, the SNR scaling factor of the reconstruction network shows sufficiently low values up from $1150 \mathrm{~Hz}$, but yields unacceptably high values for PRF values above $1400 \mathrm{~Hz}$ (cf. Fig. 19, solid line). To operate the system in these PRF ranges, the gathered data are processed in a different way: The signals of six adjacent subapertures, respectively, are processed by the multi-aperture reconstruction algorithm, yielding two output channels that are weighted and combined to maximize the signal power. Changing the order of the processing networks, this is equivalent to combining two adjacent receiving elements by the beam-steering, respectively, yielding six channels that are reconstructed unambiguously in the second stage. A further interpretation arises, if the beam-steering is combined with the multi-aperture reconstruction to a single filter function for each channel. In this case two systems of six elements each are obtained, where the respective processing is equivalent to the multi-aperture reconstruction if the systems are considered separately. The reconstructed signals from these systems are simply added afterwards.

Due to the adapted processing the number of phase centers is reduced and consequently the optimum PRF is shifted to $1440 \mathrm{~Hz}$. Further, mutual correlations cause the noise to rise. This is compensated by the increased signal power due to the doubled area of each subaperture, finally leading to a SNR scaling factor $\Phi_{b f}$ that is improved by $0.35 \mathrm{~dB}$ when comparing the values at the respective optimum PRFs (cf. Fig. 19, dashed line). Due to the reduced effective sampling, this curve starts only at $\mathrm{PRF}=B_{D} / 6$. As the azimuth 


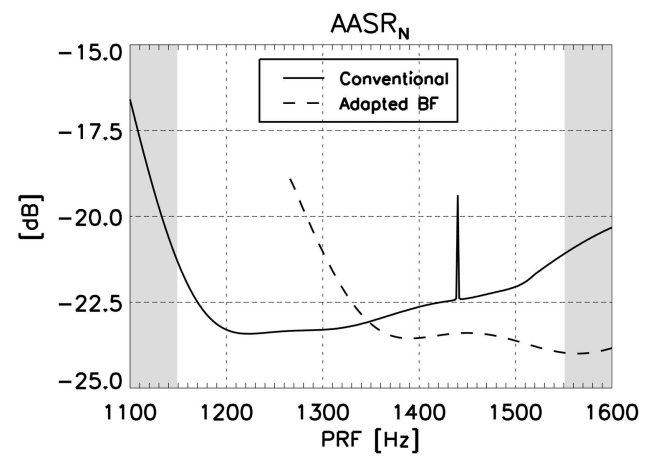

Fig. 20. $\mathrm{AASR}_{N}$ for adapted beamforming (dashed) compared with conventional DBF (solid) $\left(B_{D}=7.6 \mathrm{kHz}\right)$. Peak in conventional approach occurs at "singular" PRF at $1440 \mathrm{~Hz}$ (cf. Section IIC).

loss remains the same due to the beam-steering, the NESZ of this system can be deduced from the NESZ derived in Section C in combination with the modified $\Phi_{b f}$ of Fig. 19. Additionally, a "loss" factor of $6 / 7$ has to be considered, as the number of effective channels decreased from $N=7$ to $N=6$ (cf. (40)).

Next, the $\mathrm{AASR}_{N}$ of the system is investigated and presented in Fig. 20. The solid line shows the suppression of the original system that is improved by the adapted beamforming above $1340 \mathrm{~Hz}$ (dashed line). In combination with Fig. 19, one can conclude that the conventional approach is suitable below $1340 \mathrm{~Hz}$, while the adapted beamforming is favourable for PRF values above.

Regarding the resolution, the adapted beamforming yields a value of $1.04 \mathrm{~m}$ similar to the conventional case.

\section{DISCUSSION}

The paper summarized the state-of-the-art for SAR systems and principles that enable HRWS imaging and recalled the basic properties of multi-aperture SAR signals regarding the spatial sampling. Then, an algorithm for $\mathrm{DBF}$ on receive in multi-aperture SAR systems was presented that allows for the unambiguous recovery of the azimuth signal even in case of a nonuniformly sampled synthetic aperture, thereby avoiding any stringent PRF restriction. This multi-aperture reconstruction algorithm combines the individual receiver signals in a linear space-time processing and can be interpreted as solving a linear system of equations in a way that ambiguous parts in the azimuth signal are canceled. In a next step, the algorithm was investigated in detail with respect to the influence of the processing in the DBF network on signal power, ambiguities, and noise, yielding analytical expressions for the estimation of residual ambiguities and the determination of the SNR scaling factor $\Phi_{b f}$ of a beamforming network. This analysis showed that especially strong nonuniform sampling of the azimuth signal may cause a degraded SNR due to an increased network SNR scaling factor and an amplification of residual azimuth ambiguities. The theory was verified in simulations when evaluating an example system that was designed to image a swath of $100 \mathrm{~km}$ with a geometric resolution of $1 \mathrm{~m}$. Concerning the performance parameters, simulations were carried out including a comparison of the reconstruction algorithm to alternative methods of processing the azimuth signal. The results showed that only the reconstruction algorithm and the very similar null-steering technique provide high resolution in combination with efficient ambiguity suppression. In a next step, the main error sources that may disturb the output signal were identified to be aliased parts in the received signal and the amplification of errors and noise in the input signal due to the processing in the case of nonuniformly sampled data. Based on this analysis, optimization strategies as pattern tapering on transmit (Section VB), prebeamshaping on receive (Section VC) and adapted beamforming networks (Section VD) were introduced and the resulting performance improvement demonstrated. Pattern tapering on transmit enables an efficient suppression of ambiguous energy by adapting the pattern to the processed Doppler bandwidth and thus limiting the aliased part in the signal. In an extension of an advanced transmit architecture, an adaptive adjustment of the transmit phase center to the PRF is possible by letting the phase center "slide" over the whole antenna from pulse to pulse. Prebeamshaping on receive networks can enforce the limitation of the Doppler spectrum by tapering the receive pattern. Furthermore, such networks allow for adaptively adjusting the phase center positions to the PRF thus optimizing the spatial sampling. As a consequence, the system can be operated in a wider PRF range. Finally, the adapted beamforming method established the idea of pushing the processing from a pure suppression of ambiguous energy to a trade-off between SNR and ambiguity suppression by combining a beam-steering approach with the reconstruction algorithm. In the case of prebeamshaping on receive networks and the adapted beamforming approach, the theoretical examination of residual errors, ambiguities and the SNR scaling factor was extended to the class of cascaded beamforming networks. The extensions consider mutual correlations between different channels in contrast to conventional beamforming systems.

The focus of future work is turned to distributed SAR systems with multiple receiver satellites forming a sparse array SAR system. In principle the reconstruction algorithm is also applicable to those systems, but new aspects of importance arise that have to be considered. Firstly, the increased along-track separations of the receiving apertures have to be taken into account as they entail a high sensitivity of the sampling against PRF variations due to modulo influence on the effective sample position. On the one hand, this might cause problems, as small PRF changes have a huge impact on the 
sampling scenario, but on the other hand this offers a big opportunity for optimizing the spatial sampling by adapting the PRF as was mentioned in Section VA and Appendix D. Further, large along-track displacements might require a steering of the antenna footprints to illuminate a joint area on ground resulting in a different squint of the satellites and thus the reception of mutually shifted Doppler spectra in the receivers. This might lead to a subsampling and require new processing approaches, e.g. the combination of multi-aperture reconstruction and superresolution techniques [37]. In this context, the investigation of the impact of different antenna gains versus Doppler frequency on the processing will also be of interest. Secondly, any cross-track separation of the receivers will introduce an additional phase in the received signals, which has to be compensated, e.g., via the simultaneous acquisition of a digital elevation model in case of multiple satellites. Further, a sparse array configuration has to be carefully investigated with respect to a two-dimensional processing approach as different sensors might receive the signal of the same target with different ranges and range cell migrations as introduced in [28].

Further potential fields of application for DBF are e.g. the application of the ScanSAR or the TOPS-SAR principle in multi-aperture systems, enabling ultrawide swaths. In this context the impact of the multi-aperture reconstruction algorithm when operated in combination with burst modes is to be investigated with respect to the particular properties of such modes. As the scaling of the ambiguous energy is strongly dependent on the Doppler sub-band, the use of different subspectra for different targets in a ScanSAR or TOPS-SAR mode will result in a large variation of the residual azimuth ambiguities of these targets. So, in ScanSAR operation and even in the TOPS-SAR mode, this can result in a scalloping-like effect for the azimuth ambiguities, which is currently investigated in detail.

\section{APPENDIX A. CASCADED BEAMFORMING NETWORKS}

In the context of system optimization more sophisticated beamforming approaches are regarded as powerful tools to adaptively improve the system performance. Sections VC and VD established the idea of cascaded beamforming networks and investigated the new concept with respect to the network capability to adaptively position the systems' phase centers. In the following, the impact on signal, ambiguities and noise power that were only mentioned briefly in Section VC are derived in more detail.

\section{A. Extended System Model}

Based on the system model presented in Section IIIF and on the block diagram given in Fig. 13, we obtain new system models that are

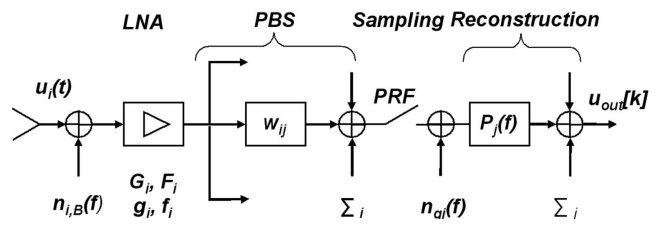

Fig. 21. Multi-aperture system model taking into account network to perform prebeamshaping on receive before signals are sampled and pass through reconstruction filter network.

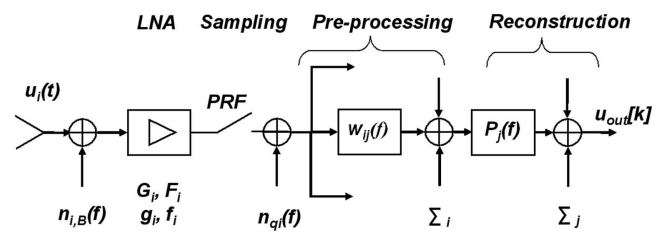

Fig. 22. Multi-aperture system model including cascaded networks to perform preprocessing after the signals are sampled and before they pass through reconstruction filter network.

extended by a block representing the additional processing steps, either a prebeamshaping (PBS) network situated before the analog-to-digital converter (ADC) (cf. Fig. 21) or a network processing the already digitized data. In the case where the preprocessing network is located behind the ADC, the order of the applied filters can of course be changed as long as the overall transfer function of each branch remains the same. The weight functions are denominated by $w_{i j}$ that (although not necessary) may be frequency dependent in the case of a filtering after the ADC (cf. Fig. 22). We start with a linear system of $K$ channels $i$ with an input signal $u_{i}(t)$, a receiver noise component $n_{i, B}(f)$, and the corresponding signal-to-noise ratio $\mathrm{SNR}_{e l, i}$, respectively. As before, receiver noise is considered to be the dominating thermal noise source and is assumed to be additive, white, and mutually uncorrelated between the channels. After reception, the signal is amplified before the $K$ input signals are weighted and combined to form $N$ intermediate output signals, each representing a virtual channel for the reconstruction filter network. These channels are then (either before or after the preprocessing) subsampled in azimuth with a ratio of PRF inducing a quantization error that is modelled as additive noise sources $n_{q j}(f)$ and $n_{q i}(f)$, respectively. Then filtering by the reconstruction filters $P_{j}(f)$ takes place followed by coherent combination of all $N$ output branches yielding the signal $u_{\text {out }}[k]$ (cf. Fig. 21, Fig. 22).

\section{B. Compensation for Power Loss}

In the analogue representation of a preprocessing network, amplitude imbalances might occur as not all elements' signals are to be split equally. Hence, depending on the necessary branching of the respective signal, the gain loss is different and has to 
be compensated to ensure uniform amplitude of all signals when entering the first processing network. This can be achieved by including the compensation in the weighting functions of this first stage by replacing the amplitude gain $\left|w_{i j}\right|$ by the new factor $\left|w_{i j}^{\prime}\right|$ according to (52), where $N_{c h, i}$ represents the number of signals in which the original signal of element $i$ is split. By this, the amplitude of the signal after being split is recovered as given in (53), where $U_{i \text {,split }}(f)$ gives the signal after splitting while $U_{i}(f)$ denotes the original signal of channel $i$. With these adapted weighting functions all following equations remain valid

$$
\begin{aligned}
\left|w_{i j}^{\prime}\right| & =\left|w_{i j}\right| \cdot \sqrt{N_{c h, i}} \\
U_{i, \text { split }}(f) \cdot w_{i j}^{\prime}(f) & =\frac{U_{i}(f)}{\sqrt{N_{c h, i}}} \cdot w_{i j}^{\prime}(f)=U_{i}(f) \cdot w_{i j}(f) .
\end{aligned}
$$

\section{Signal Power}

The desired signal that is relevant for the output signal power is determined by the input signal of the reconstruction network (cf. Section IIID) and does not depend on the location of the ADC. In contrast to the case where no prebeamshaping is applied, this signal is given by $U_{c, j}(f)$ that forms the input of the reconstruction filter network and is determined by the weighted sum of signals $U_{i}(f)$ received by the single elements and respective weighting coefficients

$$
U_{c, j}(f)=\sum_{i=1}^{K} G_{i} \cdot U_{i}(f) \cdot w_{i j}(f) .
$$

As derived in Section IIIA, $U_{i}(f)$ can be modelled as an equivalent monostatic function $U(f)$ filtered with an appropriate function that corresponds basically to a time-shift and the addition of a constant phase. To avoid confusion with the reference functions $H_{j}(f)$ that characterize the virtual channels, we denominate these functions $M_{i}(f)$. We obtain (again assuming identical LNAs in all channels and taking into account (52) if analogue preprocessing is applied) the following, where $U(f)$ represents the signal received by a single element at the same position as the transmit aperture

$$
\begin{aligned}
U_{c, j}(f) & =\sum_{i=1}^{K} G_{i} \cdot U_{i}(f) \cdot w_{i j}(f) \\
& =G \cdot U(f) \cdot \sum_{i=1}^{K} M_{i}(f) \cdot w_{i j}(f) .
\end{aligned}
$$

In a next step we assume the $w_{i j}(f)$ being the same for every virtual channel $j$ which means that all resulting virtual channels $j$ are identical in terms of their envelope and only shifted relative to each other with respect to their phase centers. This interpretation allows for expressing the signals by a equivalent monostatic signal $U_{c}(f)$ and functions $H_{j}(f)$ characterizing the virtual channels $j$ as done in (12). We can simplify (55)-(56), where $U_{c}(f)$ corresponds to the signal that arises by the weighted sum of the single elements, if the resulting phase center was situated at the same position as the transmitter. The LNA's power gain $G$ is mentioned separately as it will cancel later

$$
U_{c, j}(f)=G \cdot U_{c}(f) \cdot H_{j}(f) .
$$

Only the prebeamshaping changes the characteristic of $U_{c}(f)$ while the DBF network that reconstructs the signal only introduces a scaling factor of $N$ without modifying the pattern of $U_{c}(f)$. Hence, the signal power is determined by the spectral appearance of $U_{c}(f)$ given by the resulting pattern characteristic of the combined subapertures and the number of virtual channels $N$. Using (57), we obtain the following output signal after reconstruction in the data (cf. (58)) and in the image after focusing (cf. (59)) that correspond to (45) and (46), respectively

$$
\begin{aligned}
\left|U_{c}(f)\right| & =\left|U(f) \cdot \sum_{i=1}^{K} M_{i}(f) \cdot w_{i j}(f)\right| \\
& =A(f) \cdot\left|\sum_{i=1}^{K} M_{i}(f) \cdot w_{i j}(f)\right| \\
p_{s, c} & =N^{2} \cdot G \cdot \mathbf{E}\left[\left|U_{c}(f) \cdot \operatorname{rect}\left(f / I_{S}\right)\right|^{2}\right] \\
& =p_{s, e l} \cdot N^{2} \cdot G \cdot \mathbf{E}\left[\left|\sum_{i=1}^{K} M_{i}(f) \cdot w_{i j}(f)\right|^{2}\right] \\
p_{s, c, B_{D}} & =N^{2} \cdot G \cdot \mathbf{E}\left[\left|U(f) \cdot \sum_{i=1}^{K} M_{i}(f) \cdot w_{i j}(f) \cdot \operatorname{rect}\left(f / B_{D}\right)\right|^{2}\right] .
\end{aligned}
$$

\section{Residual Reconstruction Error}

If a preprocessing network is used before reconstruction is applied, the power of the ambiguities is to be adapted to the prebeamshaping scenario. This means that all expressions derived in Section IIIE can be adapted to the extended processing network by adding the gain factor $\mathrm{G}$ and replacing $U(f)$ by the resulting output signal of the prebeamshaping network $U_{c}(f)$ defined by (56), that represents the spectral appearance of the signal before the reconstruction network, but takes into account the preprocessing (either prebeamshaping on receive or adapted beamforming). Assuming that all virtual channels that enter the reconstruction filter network are identical in terms of weighting of the elements that form the channel, $U_{c}(f)$ is determined by the weighted 
sum given in (56) but does not include the gain $G$ of the LNA.

E. SNR Scaling of Cascaded Beamforming Networks- $\Phi_{b f, c}$

Let us consider the influence of the prebeamshaping network on the noise power under the assumption of a lossless network of weighting elements $w_{i j}(f)$. As mentioned above, the receiver noise is assumed to be mutually uncorrelated additive white Gaussian noise $n_{i, B}(f)$ with a power spectral density of $N_{0, B}$ with respect to the system bandwidth $B$. Further, quantization noise is assumed to be uniformly distributed and spectrally white as proposed in [40]. Again identical elements $i$, implying the same characteristics for $G_{i}, F i, g_{i}, f_{i}$ for all $i$ are assumed, yielding the following output noise power $p_{n, c}$ of the system

$$
\begin{aligned}
p_{n, c}= & \underbrace{p_{n, e l} \cdot G \cdot F \cdot \sum_{i=1}^{K} \mathbf{E}\left[\left|\sum_{j=1}^{N} P_{j}(f) \cdot w_{i j}(f)\right|^{2}\right]}_{\text {Receiver noise } p_{n, c, r x}} \\
& +\underbrace{p_{n, q}}_{\text {Quantization noise }} \\
= & p_{n, c, r x}+p_{n, q} .
\end{aligned}
$$

This means that the input noise power is amplified by the prebeamshaping and the following reconstruction and weighted quantization noise is added. Further, in contrast to a mere DBF network as discussed before, the mutual correlations introduced by the prebeamshaping do not allow any more for simplifying the squared sum that gives the thermal output noise power to a sum of squares. This amplification of the receiver noise is independent of the system, while the quantization error depends on the location of the ADC. If the preprocessing is applied before digitization, the quantization noise at the output is given by (61) while (62) is valid if the quantization error is introduced in the signal before the preprocessing network is passed

$$
\begin{aligned}
& p_{n, q, a}=\mathbf{E}\left[\left|\sum_{j=1}^{N} P_{j}(f) \cdot n_{q j}(f)\right|^{2}\right] \\
& p_{n, q, d}=\sum_{i=1}^{K} \mathbf{E}\left[\left|\sum_{j=1}^{N} P_{j}(f) \cdot w_{i j}(f) \cdot n_{q i}(f)\right|^{2}\right] .
\end{aligned}
$$

Again, the number of bits is assumed to be chosen such that the quantization error in the output signal will be negligible compared with the (thermal) receiver noise and consequently the overall noise is approximated by $p_{n, c, r x}$. Analogously to (34) that gives the SNR scaling factor of conventional beamforming networks, the SNR scaling factor of the cascaded network with respect to the data $\Phi_{b f, c}$ (cf. (63)) is determined by expression (58) (that gives the output signal power) and the first term of (60) that approximates the noise power. As before, the SNR scaling factor for the focused image cannot be given explicitly, as the relation between the input and output signal power depends on the shape of $U(f)$ and other parameters as defined in (59). Of course $\Phi_{b f, c}$ can be calculated using (59) for the signal power and expression (64) giving the remaining the noise power after focusing

$$
\begin{aligned}
\Phi_{b f, c} & =\frac{p_{n, c} / p_{n, e l}}{p_{s, c} / p_{s, e l}} \cdot \frac{K}{F} \\
& =\frac{K \cdot \sum_{i=1}^{K} \mathbf{E}\left[\left|\sum_{j=1}^{N} P_{j}(f) \cdot w_{i j}(f) \cdot \operatorname{rect}\left(f / I_{S}\right)\right|^{2}\right]}{N^{2} \cdot \mathbf{E}\left[\left|\sum_{i=1}^{K} M_{i}(f) \cdot w_{i j}(f) \cdot \operatorname{rect}\left(f / I_{S}\right)\right|^{2}\right]} \\
p_{n, c, B_{D}} & =p_{n, e l} \cdot G \cdot F \cdot \sum_{i=1}^{K} \mathbf{E}\left[\left|\sum_{j=1}^{N} P_{j}(f) \cdot w_{i j}(f) \cdot \operatorname{rect}\left(f / B_{D}\right)\right|^{2}\right] .
\end{aligned}
$$

\section{APPENDIX B. MULTI-APERTURE RECONSTRUCTION IN THE FRAME OF CLASSICAL STAP}

Consider, according to [47], a multi-channel beamforming system consisting of $N$ channels. Each channel $i$ is described by its input signal $s_{i}$, noise component $n_{i}$, and weighting function $a_{i}$ that are summarized by $\underline{s}=\left[s_{1}, \ldots, s_{N}\right]^{T}, \underline{n}=\left[n_{1}, \ldots, n_{N}\right]^{T}$ and $\underline{a}=\left[a_{1}, \ldots, a_{N}\right]^{T}$, respectively. The output $y$ of the system can then be described by (65), where the operator $\circ$ denotes the scalar product

$$
y=\underline{a} \circ \underline{s}+\underline{a} \circ \underline{n} .
$$

Further, if we assume an incident plane wave and use quasi-parallel approximation, each $s_{i}$ can be related to $s_{1}$ by a phase term that accounts for the azimuth angle-dependent path difference between receivers $i$ and 1 . Hence, $s$ simplifies to a steering vector, where $\theta$ gives the azimuth angle and $\Delta x_{i}$ denotes the distance of receiver $i$ to receiver 1 . Without restriction of generality we assume $\Delta x_{1}=0$ and finally obtain

$$
\underline{s}=\left[1, \exp \left[j \frac{2 \pi}{\lambda} \sin (\Theta) \Delta x_{i}\right] \ldots, \exp \left[j \frac{2 \pi}{\lambda} \sin (\Theta) \Delta x_{N}\right]\right]^{T} .
$$


If coloured noise is assumed, the optimum beamforming weights $\underline{a}$ to minimize the noise power are defined by

$$
\underline{a}^{*}=\frac{\mathbf{R}^{-1} \circ \underline{s}}{k}
$$

where $*$ denotes the complex conjugate, $k$ represents a normalization factor and $\mathbf{R}$ describes the noise covariance matrix that consists of the covariance matrix of the spatially structured noise $\mathbf{R}_{c}$ and the unity matrix I representing the white noise of power $\sigma^{2}$ :

$$
\mathbf{R}=\mathbf{E}\left[\underline{n} \circ \underline{n}^{*}\right]=\mathbf{R}_{c}+\sigma^{2} \mathbf{I} .
$$

In the special case when interpreting the ambiguous Doppler frequencies as a directive interference arriving from the respective angles and further assuming that no white noise is present, i.e., $\sigma=0$, one can show that (69) holds true, if the dependency on the azimuth angle $\theta$ is replaced by Doppler frequency $f$

$$
\underline{a}^{*}(f)=\frac{\mathbf{R}(f)^{-1} \circ \underline{s}(f)}{k}=\frac{\mathbf{R}_{c}(f)^{-1} \circ \underline{s}(f)}{k}=\mathbf{P}^{*}(f) .
$$

The matrix $\mathbf{P}$ represents the reconstruction filter functions derived in Section IIIB and contains in its $i$ th line the weighting function $a_{i}$. Hence $\mathbf{P}$ offers an alternative way of deriving the optimum beamformer to suppress azimuth ambiguities that are interpreted as directive intereferences in the STAP context.

\section{APPENDIX C. ANALOGY OF NULL-STEERING AND MULTI-APERTURE RECONSTRUCTION}

Consider a linear array of $N$ receiving apertures where the distance of aperture $i$ to aperture 0 is denoted by $\Delta x_{i}$ and the look direction is designated by $\theta$ (cf. Fig. 23). By definition we set $\Delta x_{1}=0$. After receiving the incident signal $s_{i n}(\theta)$, an individual complex weighting coefficient $a_{i}$ is applied to each of the channels' signals $s_{i}(\theta)$. Finally these signals are combined coherently, what is indicated by the " $\Sigma$," yielding the output signal $s(\theta)$.

Applying the quasi-parallel approximation, the well-known array manifold vector $v$ that characterizes the signal at each receiver $i$ with respect to the signal at receiver 1 looks as follows [46]:

$$
\begin{aligned}
\underline{v}(\Theta)= & {\left[1, \exp \left(-j \frac{2 \cdot \pi}{\lambda} \cdot \Delta x_{2} \cdot \sin \Theta\right), \ldots,\right.} \\
& \left.\quad \exp \left(-j \frac{2 \cdot \pi}{\lambda} \cdot \Delta x_{N} \cdot \sin \Theta\right)\right]^{T} \\
= & {\left[v_{1}, \ldots, v_{i}, \ldots, v_{N}\right]^{T} . }
\end{aligned}
$$

Taking into account the weighting coefficients given by the vector $\underline{a}=\left[a_{1}, \ldots, a_{N}\right]^{T}$ and the subsequent coherent summation, the resulting

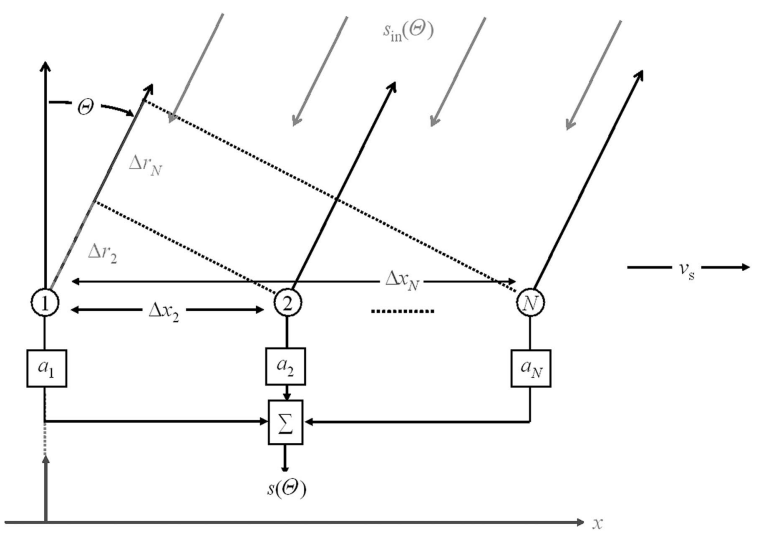

Fig. 23. Incident signal $s_{i n}(\theta)$, arriving under angle $\theta$ on linear array of $N$ receiving apertures. After reception, each receiver's signal $s_{i}(\theta)$ is weighted by $a_{i}$ and summed up yielding output signal $s(\theta)$.

output signal $s(\theta)$ is determined by (71), where $s_{1}(\theta)$ represents the signal at receiver 1

$$
\begin{aligned}
s(\Theta) & =\sum_{i=1}^{N} s_{i}(\Theta) \cdot a_{i} \\
& =s_{1}(\Theta) \cdot \sum_{i=1}^{N}\left(v_{i} \cdot a_{i}\right)=s_{1}(\Theta) \cdot \underline{v}(\Theta) \circ \underline{a} .
\end{aligned}
$$

Consider now $N$ output signals for input signals arriving from $N$ different angles $\theta_{j}, j=\{1,2, \ldots, N\}$. The respective output signals are given by the vector $s$ that can be expressed by the matrix-vector operation of (72), where $\mathbf{V}$ represents the manifold vectors for the different angles as given in (73)

$$
\begin{aligned}
s_{1}(\Theta) \cdot \mathbf{V} \cdot \underline{a} & =\underline{s} \\
\mathbf{V} & =\left[\underline{v}_{1}, \ldots, \underline{v}_{N}\right]=\left[\begin{array}{ccc}
v_{1}\left(\Theta_{1}\right) & \ldots & v_{N}\left(\Theta_{1}\right) \\
\ldots & v_{i}\left(\Theta_{j}\right) & \ldots \\
v_{1}\left(\Theta_{N}\right) & \ldots & v_{N}\left(\Theta_{N}\right)
\end{array}\right] .
\end{aligned}
$$

The weighting coefficients which are necessary to steer nulls in $N-1$ directions $\theta_{j}$ in order to "extract" the signal from the $N$ th direction are then determined by the inversion of $\mathbf{V}$. One obtains a matrix $\mathbf{A}=\mathbf{V}^{-1}$ that contains in its $k$ th column the respective weights to steer the nulls in the pattern to all directions $\theta_{j}$, $j \neq k$.

In the next step, consider a frequency $f_{1}$ and its $N-1$ ambiguous frequencies within the system azimuth bandwidth $N$. PRF, that are separated by integer multiples of PRF (cf. (74)). Using the relation between angle $\theta$ and Doppler frequency $f$ of (75), the variable $\theta_{j}$ in (73) can be replaced by the respective $f_{j}$, what yields $\mathbf{V}(f)$ in (76). Then the matrix $\mathbf{A}(f)=$ $\mathbf{V}^{-1}(f)$ contains in its $k$ th column the respective weights to steer the nulls in the pattern to all ambiguous frequencies $f_{j}, j \neq k$, while recovering $f_{k}$. 
Hence, calculating and applying $\mathbf{A}(f)$ for all $f_{1}$ within the interval $I_{1}=[-N \cdot \mathrm{PRF} / 2,-(N-2) \cdot \mathrm{PRF} / 2]$ and the respective ambiguous Doppler frequency sets in (74) allows for unambiguously recovering the original spectrum of bandwidth $N \cdot$ PRF the inter-sample distance is smaller than the inter-pulse distance. Hence in such a system, the potential is limited as small changes in the PRF do not allow for large variations in the sampling. In contrast, in sparse arrays, the spatial position of

$$
\begin{aligned}
& f_{1}+[0, \mathrm{PRF}, \ldots,(N-1) \cdot \mathrm{PRF}]=:\left[f_{1}, \ldots, f_{j}, \ldots, f_{N}\right] \quad \text { with } \quad f_{1} \in\left[-\frac{N \cdot \mathrm{PRF}}{2},-\frac{(N-2) \cdot \mathrm{PRF}}{2}\right] \\
& f(\Theta)=\frac{2 \cdot v}{\lambda} \cdot \sin \Theta \\
& \mathbf{V}(f)=\left[\begin{array}{ccc}
\exp \left[-j \cdot \frac{\pi \cdot \Delta x_{1}}{v} \cdot f\right]_{1} & \exp \left[-j \cdot \frac{\pi \cdot \Delta x_{N}}{v} \cdot f_{1}\right] \\
\cdots & \exp \left[-j \cdot \frac{\pi \cdot \Delta x_{i}}{v} \cdot\left(f_{1}+(j-1) \cdot \mathrm{PRF}\right)\right] & \ldots \\
\exp \left[-j \cdot \frac{\pi \cdot \Delta x_{1}}{v} \cdot\left(f_{1}+(N-1) \cdot \mathrm{PRF}\right)\right] & \exp \left[-j \cdot \frac{\pi \cdot \Delta x_{N}}{v} \cdot\left(f_{1}+(N-1) \cdot \mathrm{PRF}\right)\right]
\end{array}\right] .
\end{aligned}
$$

Let us now compare above shown matrix $\mathbf{V}(f)$ to the system matrix $\mathbf{H}(f)$ derived in Section III. We observe that under the assumed approximations, $\mathbf{V}(f)$ is nearly identical to $\mathbf{H}(f)$. The only difference is the constant phase term relating the bi- and monostatic sample position, that is accounted for in $\mathbf{H}(f)$ while it is neglected in $\mathbf{V}(f)$. Hence the multi-aperture reconstruction algorithm can be regarded as an extension of the classical null-steering approach that also accounts for large receiver spacing. In the case of a single-platform system, the distances between the receiving apertures are small, and consequently the resulting phase differences are negligible.

\section{APPENDIX D. ADAPTIVE PRF MANAGEMENT IN SPARSE ARRAY SYSTEMS}

As mentioned in Section VA, the conditions for the multi-aperture processing can be improved by harmonizing the phase centers with the PRF to obtain a sampling as uniform as possible. In sparse array systems, an adaptive management of the PRF shows great potential to adjust the virtual sample positions. Recalling the results derived in Section II, we can express the distance between adjacent samples originating from transmit pulse $k$ at channel $j$ and transmit pulse $l$ at channel $i$ by (77), where $\Delta x_{j}$ denotes the distance of the receiver to the transmitter

$$
\begin{gathered}
\Delta x_{j, i}=x_{j, k}-x_{i, l}=\frac{\Delta x_{j}}{2}-\frac{\Delta x_{i}}{2}+(k-l) \cdot \frac{v_{s}}{P R F}, \\
k, l \in \mathbf{Z} .
\end{gathered}
$$

In a conventionally operated single platform system, adjacent samples originate from the same or subsequent pulses, i.e., $|k-l| \leq 1$, and consequently adjacent samples is not bound to the same transmitted pulse as in single platform systems. One recognizes that variations in the PRF result in variations of the position $\Delta x_{j, i}$ amplified by a factor $(k-l)$ and hence only small changes in the PRF might already have a large impact on the spatial distribution of the samples. This offers wide flexibility to adapt the sampling even if only a small variation of the PRF is possible and allows thus for compensating for the high sensitivity of the systems' $\mathrm{SNR}$ and $\mathrm{AASR}_{N}$ regarding PRF variations.

\section{REFERENCES}

[1] Curlander, J., and McDonough, R. Synthetic Aperture Radar-Systems and Signal Processing. New York: Wiley, 1991.

[2] Carrara, W., Goodman, R., and Majewski, R. Spotlight Synthetic Aperture Radar: Signal Processing Algorithm.

Boston: Artech House, 1995.

[3] Tomiyasu, K.

Conceptual performance of a satellite borne, wide swath synthetic aperture radar.

IEEE Transactions on Geoscience and Remote Sensing, GRS-19 (1981), 108-116.

[4] Moore, R. K., Claassen, J. P., and Lin, Y. H. Scanning spaceborne synthetic aperture radar with integrated radiometer.

IEEE Transactions on Aerospace and Electronic Systems, AES-17 (1981), 410-421.

[5] De Zan, F., and Monti Guarnieri, A. M TOPSAR: Terrain observation by progressive scans. IEEE Transactions on Geoscience and Remote Sensing, 44 (2006), 2352-2360.

[6] Claassen, J. P., and Eckerman, J.

A system for wide swath constant incident angle coverage.

In Proceedings of Synthetic Aperture Radar Technology Conference, Las Cruces, NM, 1978. 
[7] Jain, A.

Multibeam synthetic aperture radar for global oceanography.

IEEE Transactions on Antennas and Propagation, AP-27 (1979), 535-538

[8] Jean, B. R., and Rouse, J. W.

A multiple beam synthetic aperture radar design concept for geoscience applications.

IEEE Transactions on Geoscience and Remote Sensing, GRS-21 (1983), 201-207.

[9] Griffiths, H., and Mancini, P.

Ambiguity suppression in SARs using adaptive array techniques.

In Proceedings of IEEE International Geoscience and Remote Sensing Symposium (IGARSS), Espoo, Finland, 1991, 1015-1018.

[10] Currie, A., and Brown, M. A.

Wide-swath SAR.

IEE Proceedings—Radar Sonar and Navigation, 139, 2 (1992), 122-135.

[11] Callaghan, G. D., and Longstaff, I. D.

Wide swath spaceborne SAR using a quad element array. IEE Proceedings-Radar Sonar and Navigation, 146, 3 (1999), 159-165.

[12] Goodman, N., Rajakrishna, D., and Stiles, J.

Wide swath, high resolution SAR using multiple receive apertures.

In Proceedings of IEEE Geoscience and Remote Sensing Symposium (IGARSS), vol. 3, Hamburg, Germany, 1999, 1767-1769.

[13] Younis, M., and Wiesbeck, W.

SAR with digital beamforming on receive only.

In Proceedings of IEEE Geoscience and Remote Sensing Symposium (IGARSS), vol. 3, Hamburg, Germany, 1999, 1773-1775.

[14] Suess, M., Grafmüller, B., and Zahn, R.

A novel high resolution, wide swath SAR system.

In Proceedings of IEEE Geoscience and Remote

Sensing Symposium (IGARSS), Sydney, Australia, 2001, 1013-1015.

[15] Suess, M., and Wiesbeck, W.

Side-looking synthetic aperture system.

EP 1241487 A 1, 2001.

[16] Wiesbeck, W.

SDRS: Software-defined radar sensors.

In Proceedings of IEEE Geoscience and Remote Sensing Symposium (IGARSS), Sydney, Australia, 2001, 3259-3261.

[17] Suess, M., Zubler, M., and Zahn, R.

Performance investigation on the high resolution, wide swath SAR system.

In Proceedings of European Conference on Synthetic Aperture Radar (EUSAR), Ulm, Germany, 2002, 187-191.

[18] Brule, L., and Baeggli, H.

Radarsat-2 program update.

In Proceedings of IEEE Geoscience and Remote Sensing Symposium (IGARSS), Toronto, Canada, 2002.

[19] Goodman, N., Lin, S., Rajakrishna, D., and Stiles, J. Processing of multiple-receiver spaceborne arrays for wide area SAR.

IEEE Transactions on Geoscience and Remote Sensing, $\mathbf{4 0 ,}$ 4 (Apr. 2002), 841-852.

[20] Krieger, G., Fiedler, H., Rodriguez-Cassola, M., Hounam, D., and Moreira, A.

System concepts for bi- and multistatic SAR missions. In Proceedings of the ASAR Workshop 2003, Saint-Hubert, Quebec, Canada, June 2003.
[21] Krieger, G., and Moreira, A.

Potentials of digital beamforming in bi- and multistatic SAR.

In Proceedings of IEEE International Geoscience and Remote Sensing Symposium (IGARSS), Toulouse, France, 2003, 527-529.

[22] Heer, C., Soualle, F., Zahn, R., and Reber, R.

Investigations on a new high resolution wide swath SAR concept.

In Proceedings of IEEE International Geoscience and Remote Sensing Symposium (IGARSS), Toulouse, France, 2003, 521-523, 2003.

[23] Younis, M., Fischer, C., and Wiesbeck, W. Digital beamforming in SAR systems.

IEEE Transactions on Geoscience and Remote Sensing, 41, 7 (July 2003), 1735-1739.

[24] Younis, M., Venot, Y., and Wiesbeck, W. A simulator for digital beam forming SAR. In Proceedings of International Radar Symposium (IRS), Dresden, Germany, 2003.

[25] Mittermayer, J., and Runge, $\mathrm{H}$.

Conceptual studies for exploiting the TerraSAR-X dual receiving antenna.

In Proceedings of Geoscience and Remote Sensing Symposium (IGARSS), Toulouse, France, 2003.

[26] Aguttes, J. P.

The SAR train concept: Required antenna area distributed over $\mathrm{N}$ smaller satellites, increase of performance by $\mathrm{N}$. In Proceedings of Geoscience and Remote Sensing Symposium (IGARSS), Toulouse, France, 2003.

[27] Krieger, G., Gebert, N., and Moreira, A. Unambiguous SAR signal reconstruction from nonuniform displaced phase center sampling. IEEE Geoscience and Remote Sensing Letters, 1 (2004), 260-264.

[28] Krieger, G., Gebert, N., and Moreira, A.

SAR signal reconstruction from non-uniform displaced phase center sampling.

In Proceedings of Geoscience and Remote Sensing Symposium (IGARSS), Anchorage, AK, Sept. 2004.

[29] Krieger, G., Gebert, N., and Moreira, A.

Digital beamforming and non-uniform displaced phase centre sampling in bi- and multistatic SAR.

In Proceedings of European Conference on Synthetic Aperture Radar (EUSAR), Ulm, Germany, 2004.

[30] Younis, M.

Digital beam-forming for high-resolution wide swath real and synthetic aperture radar.

Karlsruhe, Germany, 2004.

[31] Gebert, N., Krieger, G., and Moreira, A.

SAR signal reconstruction from non-uniform displaced phase centre sampling in the presence of perturbations. In Proceedings of Geoscience and Remote Sensing Symposium (IGARSS), Seoul, South Korea, 2005.

[32] Gebert, N., Krieger, G., and Moreira, A.

High resolution wide swath SAR imaging-System performance and influence of perturbations. In Proceedings of International Radar Symposium (IRS), Berlin, Germany, 2005.

[33] Li, Z., Wang, H., Su, T., and Bao, Z. Generation of wide-swath and high-resolution SAR images from multichannel small spaceborne SAR system. IEEE Geoscience and Remote Sensing Letters, 2, 1 (Jan. 2005), 82-86.

[34] Li, Z., Bao, Z., Wang, H., and Liao, G. Performance improvement for constellation SAR using signal processing techniques.

IEEE Transactions on Aerospace and Electronic Systems, 42, 2 (2006), 436-452. 
[35] Fischer, C., Heer, C., Krieger, G., and Werninghaus, R. A high resolution wide swath SAR.

In Proceedings of European Conference on Synthetic Aperture Radar (EUSAR), Dresden, Germany, 2006.

[36] Gebert, N., Krieger, G., and Moreira, A.

High resolution wide swath SAR imaging with digital beamforming-Performance analysis, optimization and system design.

In Proceedings of European Conference on Synthetic Aperture Radar (EUSAR), Dresden, Germany, 2006.

[37] Krieger, G., and Moreira, A.

Spaceborne bi- and multistatic SAR: Potential and challenges.

IEE Proceedings-Radar, Sonar and Navigation, 153 (2006), 184-198.

[38] Papoulis, A.

Generalized sampling expansion.

IEEE Transactions on Circuits and Systems, CAS-24, 11 (1977), 652-654.

[39] Brown, J.

Multi-channel sampling of low-pass signals.

IEEE Transactions on Circuits and Systems, CAS-28, 2 (1981), 101-106.

[40] Oppenheim, A.

Discrete-Time Processing.

Upper Saddle River, NJ: Prentice-Hall, 1999.

[41] Wang, T., and Bao, Z.

Improving the image quality of spaceborne mulitple-aperture SAR under minimization of sidelobe clutter and noise.

IEEE Geoscience and Remote Sensing Letters, 3, 3 (July 2006), 297-301.
[42] Márquez-Martínez, J., Mittermayer, J., and

Rodríguez-Cassolà, $\mathrm{M}$.

Radiometric resolution optimization for future SAR systems.

In Proceedings of Geoscience and Remote Sensing Symposium (IGARSS), Anchorage, AK, Sept. 2004.

[43] Kraus, J.

Antennas.

New York: McGraw-Hill, 1950.

[44] Krieger, G., Gebert, N., and Moreira, A.

Multidimensional waveform encoding: A new digital beamforming technique for synthetic aperture radar remote sensing.

IEEE Transactions on Geoscience and Remote Sensing, 46, 1 (2008), 31-46.

[45] Krieger, G., et al.

TanDEM-X: A satellite formation for high-resolution

SAR interferometry.

IEEE Transactions on Geoscience and Remote Sensing, 45,

11,1 (2007), 3317-3341.

[46] van Trees, H. L.

Optimum Array Processing_Part IV of Detection,

Estimation, and Modulation Theory.

New York: Wiley, 2002.

[47] Guerci, J. R.

Space-Time Adaptive Processing.

Boston: Artech House, 2003.

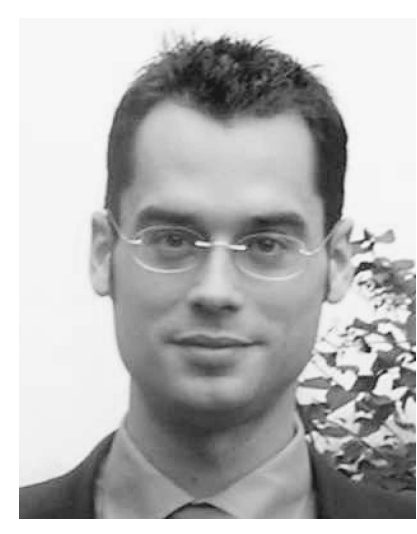

Nicolas Gebert received the Dipl.-Ing (M.S.) from the Munich University of Technology (TU München), Munich, Germany, in 2003.

$\mathrm{He}$ is currently as a Ph.D. student with the German Aerospace Center (DLR) in Oberpfaffenhofen, Germany. His major research areas are digital beamforming concepts with focus on signal processing, system design, and optimization of multi-channel SAR systems for high performance imaging applications. Further interests are the development of innovative and advanced concepts for ultra-wide-swath imaging with SAR systems. 


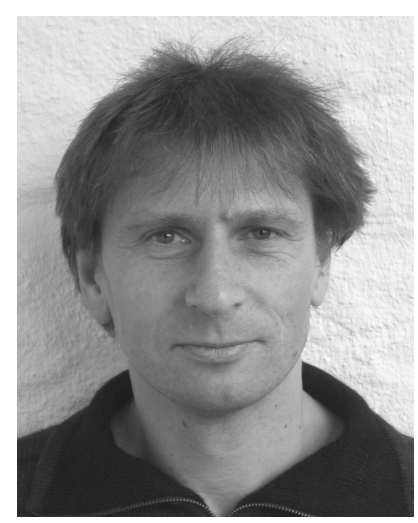

Gerhard Krieger (M'03) received the Dipl.-Ing. (M.S.) and Dr.-Ing. (Ph.D.) degrees (with honors) in electrical and communication engineering from the Technical University of Munich, Germany, in 1992 and 1999, respectively.

From 1992 to 1999, he was with the Ludwig-Maximilians University, Munich, where he worked as an interdisciplinary research scientist on the modeling of biological and technical vision systems. In 1999, he joined the Microwaves and Radar Institute (HR) of the German Aerospace Center (DLR), Oberpfaffenhofen, Germany, where he developed signal processing algorithms for an innovative forward looking radar system. From 2001 to 2007 he led the New SAR Missions Group which devised new bistatic and multistatic radar systems like the forthcoming TanDEM-X mission as well as innovative digital beamforming techniques for advanced multi-channel SAR. Since 2008, he has been Head of the new Radar Concepts Department of the Microwaves and Radar Institute, DLR, Oberpfaffenhofen, Germany. His research interests include innovative remote sensing systems and applications based on radar interferometry, tomography, biand multistatic satellite formations, digital beamforming, and the development of advanced signal and image processing algorithms.

Alberto Moreira (M'92-SM'96-F'04) was born in Sõo José dos Campos, Brazil, in 1962. He received the B.S.E.E. and the M.S.E.E. degrees, in 1984 and 1986, respectively, from the Aeronautical Technological Institute ITA, Brazil and the Eng. Dr. degree (Honors) from the Technical University of Munich, Germany, 1993.

In 2003, he received a full professorship from the University of Karlsruhe, Germany, in the field of microwave remote sensing. As its chief scientist and engineer, he managed from 1996 to 2001 the SAR Technology Department of the Microwaves and Radar Institute at the German Aerospace Center (DLR). Under his leadership, the DLR airborne SAR system, E-SAR, has been upgraded to operate in innovative imaging modes like polarimetric SAR interferometry and SAR tomography. Since 2001, he is the director of the Microwaves and Radar Institute at DLR. The Institute contributes to several scientific programs and space projects for actual and future air- and space-borne SAR missions. Recently, the mission proposal TanDEM-X lead by his Institute has been approved for the realization phase. He is the principal investigator for this mission. His professional interests and research areas encompass radar end-to-end system design and analysis, innovative microwave techniques and system concepts, signal processing, and remote sensing applications.

Dr. Moreira is serving as a member of the IEEE GRSS Administrative

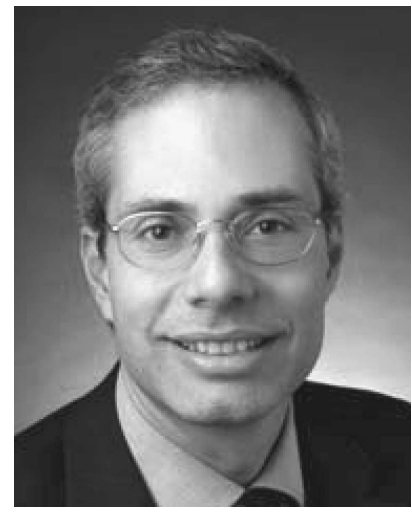
Committee (1999-2001, 2004-2006), is the chair of the German Chapter of the GRSS since 2003, and is actively serving as an associate editor for the Geoscience and Remote Sensing Letters. Since 2003 he is also serving as a member of the Board of Directors of the ITG (Information Technology Society) of VDE (German Association for Electrical, Electronic and Information Technologies). In 1995, he was the recipient of the DLR Science Award. He and his colleagues received the GRSS Transactions Prize Paper Awards in 1997 and 2001, respectively. He is also the recipient of the IEEE Nathanson Award (1999) and the IEEE Kiyo Tomiyasu Award (2007). He has contributed to the successful series of the European SAR conferences (EUSAR) since 1996 as member of the Technical Program Committee, Technical Chairman (2000), Awards Chairman (2002-2004) and General Chairman (2006). 